\title{
Supraphysiological androgens suppress prostate cancer growth through androgen receptor-mediated DNA damage
}

\author{
Payel Chatterjee, ${ }^{1}$ Michael T. Schweizer, ${ }^{2,3}$ Jared M. Lucas, ${ }^{1}$ Ilsa Coleman, ${ }^{1}$ Michael D. Nyquist, ${ }^{1}$ Sander B. Frank, ${ }^{1}$ Robin Tharakan, ${ }^{1}$ \\ Elahe Mostaghel, ${ }^{2,3}$ Jun Luo, ${ }^{4}$ Colin C. Pritchard, ${ }^{5}$ Hung-Ming Lam, ${ }^{6}$ Eva Corey, ${ }^{6}$ Emmanuel S. Antonarakis, ${ }^{7}$ Samuel R. Denmeade, \\ and Peter S. Nelson ${ }^{1,2,3}$
}

'Division of Human Biology and 'Division of Clinical Research, Fred Hutchinson Cancer Research Center, Seattle, Washington, USA. ${ }^{3}$ Department of Medicine, University of Washington, Seattle, Washington, USA. ${ }^{4}$ Department of Urology, Johns Hopkins University, Baltimore, Maryland, USA. ${ }^{5}$ Department of Laboratory Medicine and ${ }^{6}$ Department of Urology, University of Washington, Seattle, Washington, USA. 7Department of Oncology, The Sidney Kimmel Comprehensive Cancer Center, Johns Hopkins University, Baltimore, Maryland, USA

Prostate cancer (PC) initially depends on androgen receptor (AR) signaling for survival and growth. Therapeutics designed to suppress AR activity serve as the primary intervention for advanced disease. However, supraphysiological androgen (SPA) concentrations can produce paradoxical responses leading to PC growth inhibition. We sought to discern the mechanisms by which SPA inhibits PC and to determine if molecular context associates with antitumor activity. SPA produced an ARmediated, dose-dependent induction of DNA double-strand breaks, $G_{0} / G_{1}$ cell-cycle arrest, and cellular senescence. SPA repressed genes involved in DNA repair and delayed the restoration of damaged DNA, which was augmented by poly (ADPribose) polymerase 1 inhibition. SPA-induced double-strand breaks were accentuated in BRCA2-deficient patients with PC, and combining SPA with poly (ADP-ribose) polymerase or DNA-dependent protein kinase inhibition further repressed growth. Next-generation sequencing was performed on biospecimens from patients with PC receiving SPA as part of ongoing phase II clinical trials. Patients with mutations in genes mediating homology-directed DNA repair were more likely to exhibit clinical responses to SPA. These results provide a mechanistic rationale for directing SPA therapy to patients with PC who have AR amplification or DNA repair deficiency and for combining SPA therapy with poly (ADP-ribose) polymerase inhibition.

\section{Introduction}

Androgens, the androgen receptor (AR), and the AR-signaling program are intimately associated with the pathogenesis of prostate cancer (PC) (1-3). Suppressing AR signaling through androgen-deprivation therapy (ADT) was determined to be an effective approach for treating patients with advanced PC in the 1940 s and remains a key component of current treatment regimens (4). Despite substantial initial responses, metastatic PC almost universally develops resistance to ADT, leading to a clinical state termed castration-resistant prostate cancer (CRPC). The emergence of CRPC is generally accompanied by a revival of AR signaling (5-7).

Authorship note: PC, MTS, and JML contributed equally to this work. SRD and PSN contributed equally to this work.

Conflict of interest: PSN is a paid consultant/advisor to Janssen and Astellas. MTS is a paid consultant/advisor to Janssen and has received research funding for The University of Washington from Janssen, AstraZeneca, Zenith, Pfizer, and Hoffmann-La Roche. ESA is a paid consultant/advisor to Janssen, Astellas, Sanofi, Dendreon, ESSA, AstraZeneca, Amgen, Clovis, and Merck; he has received research funding for Johns Hopkins University from Janssen, Johnson \& Johnson, Sanofi, Dendreon, Genentech, Novartis, Bristol Myers-Squibb, AstraZeneca, Celgene, Clovis, and Merck. ESA and JL are coinventors of a biomarker technology that has been licensed to Qiagen. Copyright: ( 2019, American Society for Clinical Investigation.

Submitted: January 22, 2019; Accepted: July 11, 2019; Published: September 4, 2019

Reference information: J Clin Invest. 2019;129(10):4245-4260.

https://doi.org/10.1172/JCl127613.
Collectively, current data indicate that the AR remains a viable target for the treatment of most men with metastatic CRPC.

Although the vast majority of therapeutic strategies directed toward the AR pathway are designed to inhibit signaling, there is long-standing evidence that a subset of PCs are repressed by the administration of testosterone $(\mathrm{T})$, particularly after long periods of adaptation to growth in a low androgen environment (8-11). Abundant data from preclinical models reproducibly demonstrate biphasic responses, whereby at physiological androgen concentrations proliferation is induced, but at high supraphysiological androgen (SPA) concentrations, growth is suppressed $(8,9,12)$. Similar effects have been observed in patients with breast cancer exposed to high concentrations of estrogen $(13,14)$. Studies of PC metastases have shown inverse relationships between AR expression and measures of cell proliferation (15), and recent prospective clinical trials of SPA have demonstrated prostate-specific androgen (PSA) declines and tumor regression in subsets of men with CRPC $(10,11)$. Consequently, understanding the mechanisms underlying the growth-repressive effects of SPA may serve to determine individuals optimally treated by this approach and identify drug combinations that could synergize with SPA to produce more substantial and durable responses.

To date, several mechanisms have been postulated to underlie SPA-mediated growth repression including suppressing cMyc activity, upregulating negative cell-cycle regulators such as p27, and impairing DNA licensing required for cycles of DNA replication (16- 
A

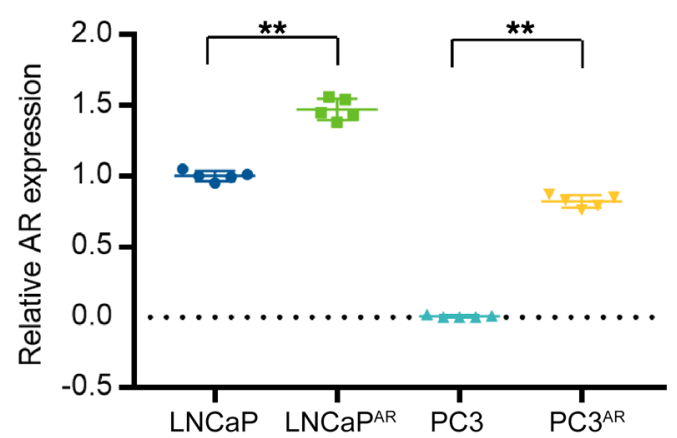

B

LNCaP
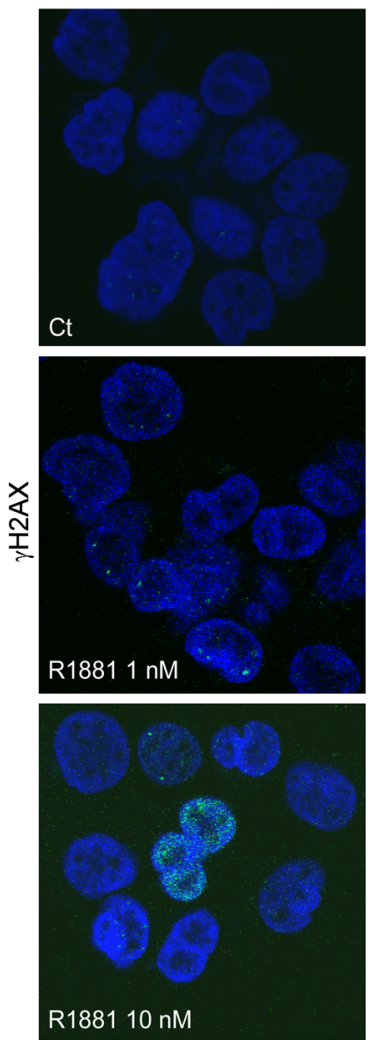

C

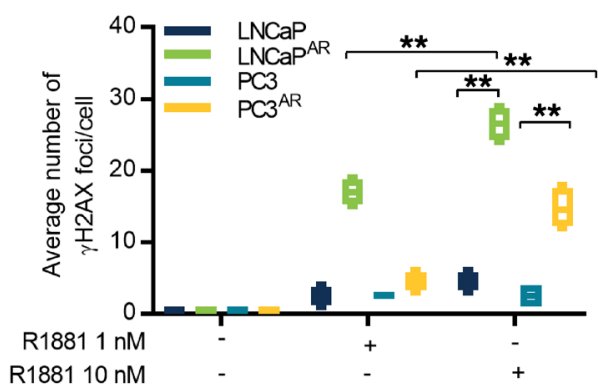

D

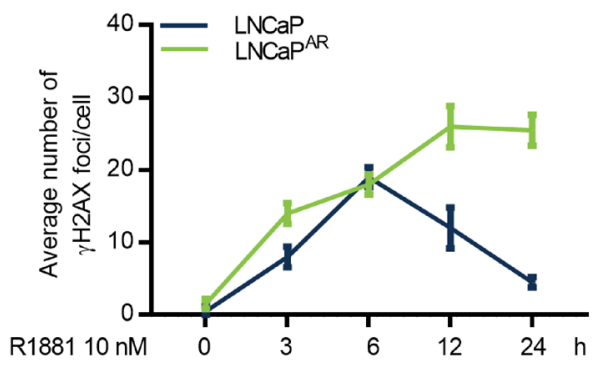

E
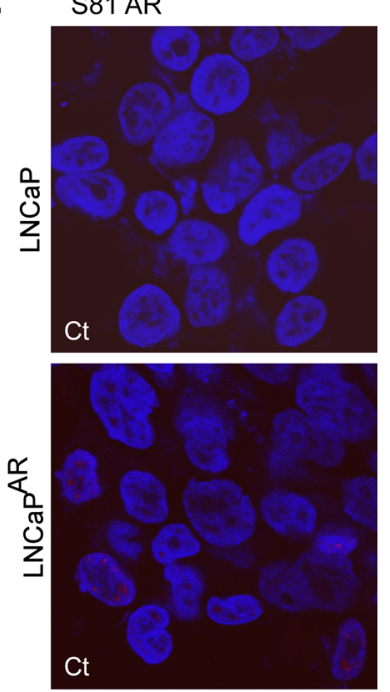

$\mathbf{F}$

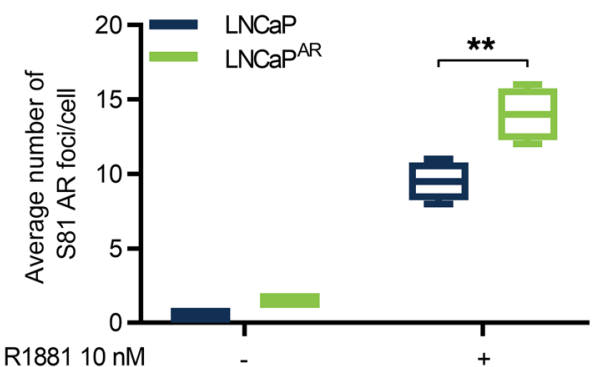

G

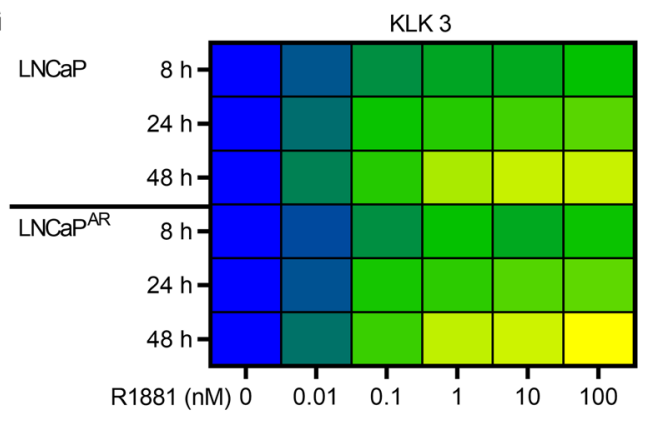

$\mathrm{NK} \times 3.1$

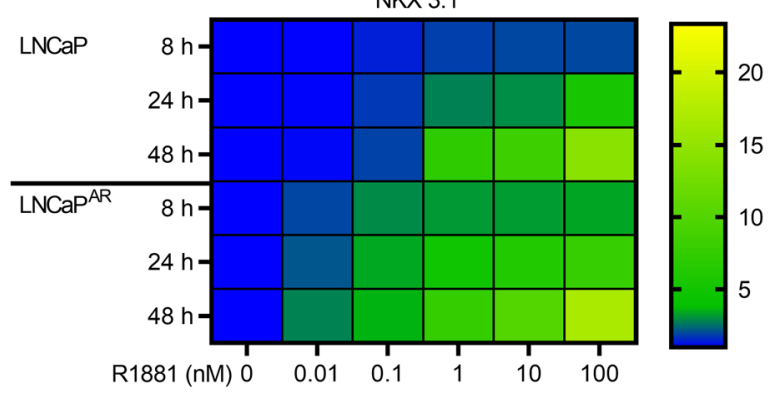

TMPRSS2

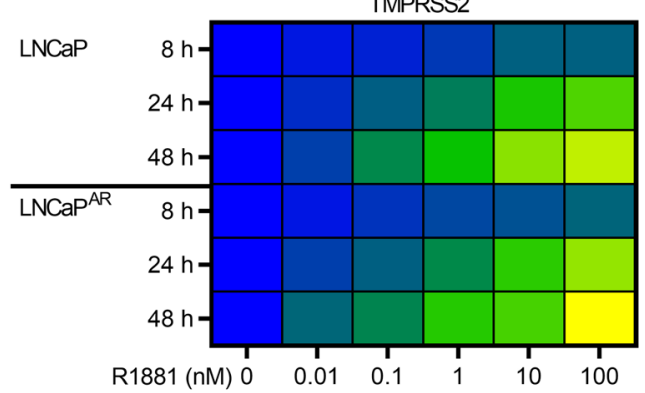


Figure 1. Supraphysiological androgen concentrations promote DNA damage and enhance AR transcriptional output. (A) qRT-PCR quantitation of AR transcript levels $(n=5)$. (B) Confocal immunostaining of $\gamma \mathrm{H} 2 \mathrm{AX}$ in $\mathrm{LNCaP}$ and $\mathrm{LNCaP}{ }^{\mathrm{AR}}$ cells in normal growth medium, control medium (Ct), or 24 hours after treatment with $1 \mathrm{nM}$ or $10 \mathrm{nM}$ R1881. (C) Quantitation of $\gamma \mathrm{H} 2 \mathrm{AX}$ foci in all cell lines exposed to $1 \mathrm{nM}$ and $10 \mathrm{nM}$ R1881. Average number of foci were plotted by calculating the mean FPC from different fields. (D) Quantitation of $\gamma \mathrm{H} 2 \mathrm{AX}$ foci measured in LNCaP and LNCaPAR cells at time intervals following exposure to $10 \mathrm{nM}$ R1881. (E) Immunofluorescence analysis of AR S81 phosphorylation in LNCaP cells in control medium (Ct) or $10 \mathrm{nM}$ 1881. (F) Quantitation of AR 581 foci in LNCaP and LNCaPAR cells. (C) Transcript levels of KLK3, NKX3.1, and TMPRSS2 in LNCaP cells following exposure to R881 concentrations $0.01-100 \mathrm{nM}$. In C, D and F, data represent the mean $\pm S D$ ( $n=3$ replicates per experiment). Original magnification for $\mathbf{B}$ and $\mathbf{E}: \times 40$. ${ }^{* *} P<0.01$ by 2 -way ANOVA.

18). Notably, T has been shown to induce DNA damage and promote the antitumor effects of ionizing radiation $(19,20)$. This observation is consistent with studies of regulated transcription by nuclear hormone receptors whereby receptor activation induces transient DNA double-strand breaks (DSBs) at enhancer and promoter regulatory sites in order to facilitate gene expression by releasing DNA topologies that constrain RNA polymerase function $(19,21,22)$.

In the context of therapeutics, preclinical studies have demonstrated a paradox: although DNA repair genes may be regulated by AR, both ADT and SPA augment the effects of radiation-induced DNA damage and improve antitumor responses (20, 23, 24). The AR is recruited to enhancer and promoter regions of several DNA damage repair genes and directly enhances their transcription (23-25). Reducing AR activity by ligand deprivation or AR antagonism downregulates the expression of these DNA damage repair genes and synergizes with ionizing radiation to induce DNA DSBs, reduce DNA repair responses, and augment cell killing (23, 24). The impairment of AR-mediated DNA repair is postulated to underlie the enhanced survival benefit observed in patients concurrently treated with localized radiation therapy and ADT.

In the present study, we sought to determine the mechanisms and the context by which SPA optimally exerts antitumor effects. We confirmed previous findings demonstrating that SPA induces DNA DSBs in the setting of AR expression and found that elevated AR levels, as observed in a subset of men treated with ADT and progressing to CRPC, potentiate SPA-induced DSBs and rates of apoptosis. Further, PC cell lines or patient-derived xenografts (PDXs) deficient in BRCA2 exhibit elevated DNA damage and cell death when exposed to SPA. The pharmacological inhibition of poly (ADP-ribose) polymerase 1 (PARP1) or DNA-dependent protein kinase (DNA-PKcs) augmented these SPA effects. In support of these observations, patients with metastatic CRPC with mutations in genes mediating homology-directed DNA repair were more likely to exhibit clinical responses to SPA. These results support specific clinical strategies designed to optimize the use of SPA for the treatment of men with CRPC.

\section{Results}

Supraphysiological concentrations of androgens induce DNA damage in PCs expressing the androgen receptor. To determine the relationships between the $\mathrm{AR}$, androgens, and the induction of DNA damage, specifically DSBs, we evaluated the effects of SPA on PC cell lines with or without native AR activity (LNCaP and $\mathrm{PC} 3$, respectively) or cells engineered to express high levels of $\mathrm{AR}, \mathrm{LNCaP}^{\mathrm{AR}}$, and PC3 ${ }^{\mathrm{AR}}$ (Figure 1A). We treated these cells with a range of androgen concentrations and measured DNA damage, senescence, and apoptotic responses. Normal eugonadal serum concentrations of $\mathrm{T}$ and the high-affinity $\mathrm{T}$ metabolite $5-\alpha$ dihydrotestosterone (DHT) are about $17 \mathrm{nM}(500 \mathrm{ng} / \mathrm{dL})$ and approximately $1 \mathrm{nM}$, respectively, for men aged 60 years, with DHT approximately 5 -fold more potent than $\mathrm{T}$ with respect to activating the $\operatorname{AR}(26,27)$. In men treated with surgical or medical ADT, serum concentrations of T and DHT are approximately $1 \mathrm{nM}$ and 0.1-0.5 nM, respectively (27). We used DHT, or the synthetic androgen methyltrienolone/R1881, as it exhibits AR binding affinities equivalent to DHT and unlike DHT is nonaromatizable. We exposed PC cells to charcoal-stripped growth medium depleted of androgens, to reflect castrate conditions, $1 \mathrm{nM} \mathrm{DHT/}$ R1881 to reflect eugonadal concentrations, or $10 \mathrm{nM}$ DHT/R1881 to reflect SPA concentrations. We quantified DNA DSBs by comet assays and by confocal immunofluorescence staining for phosphorylated $\mathrm{H} 2 \mathrm{AX}(\gamma \mathrm{H} 2 \mathrm{AX})$ and 53BP1.

Treatment with R1881 or DHT resulted in concentrationdependent increases in $\gamma \mathrm{H} 2 \mathrm{AX}$ and 53BP1 foci in AR-expressing cells (Figure 1, B and C; and Supplemental Figure 1, A-E; supplemental material available online with this article; https:// doi.org/10.1172/JCI127613DS1). Very rare foci were observed in AR-null PC3 cells (Figure 1C and Supplemental Figure 1, B, $\mathrm{D}$, and $\mathrm{E})$. LNCaP cells engineered to express AR approximately $50 \%$ above the endogenous levels exhibited substantially higher numbers of DSBs at $1 \mathrm{nM}$ R1881: 16 foci per cell (FPC) compared with 2 FPC $(P<0.01)$, which increased further with higher R1881 concentrations $(P<0.01)$ (Figure $1, \mathrm{~B}$ and $\mathrm{C})$. AR-positive VCaP cells also showed increases in $\gamma \mathrm{H} 2 \mathrm{AX}$ foci following SPA treatment (Supplemental Figure 2, A and D). The introduction of AR into PC3 cells resulted in substantial increases in $\gamma \mathrm{H} 2 \mathrm{AX}$ and 53BP1 following exposure to androgens, and the foci numbers increased with higher androgen concentrations (Figure 1C and Supplemental Figure 1, B, D, and E). Comet assays demonstrated evidence of DNA damage in both LNCaP and LNCaPAR cells following SPA treatment: the tail moment and length both were significantly increased at 6 hours compared with controls and persisted up to 24 hours in LNCaPAR cells (Supplemental Figure 1, I and J). We also evaluated AR-null DU145 cells and LNCaP cells engineered to eliminate AR expression (LNCaP ${ }^{\mathrm{APIPC}}$ ) (28). No increase in $\gamma \mathrm{H} 2 \mathrm{AX}$ foci were observed in either line after exposure to R1881 (Supplemental Figure 2, B and C), confirming that the AR is required for the androgen-induced generation of DNA DSBs.

The level of AR also influenced the kinetics of DSB resolution. The number of $\gamma \mathrm{H} 2 \mathrm{AX}$ foci as well as comet tail length and moment peaked at 6 hours after exposure to R1881 or DHT in parental LNCaP cells with endogenous AR expression, and the foci declined to near baseline levels by 24 hours (Figure 1D and Supplemental Figure 1, F, I, and J). In LNCaP ${ }^{A R}$ cells, the number of foci further increased at 12 hours and persisted at 24 hours. Similar results were observed in PC3 ${ }^{\mathrm{AR}}$ and with DHT (Supplemental Figure 1, G and $\mathrm{H}$ ). The time difference in foci persistence after R1881 versus DHT treatment could be explained by the rapid metabolism of DHT compared with R1881 $(29,30)$. 

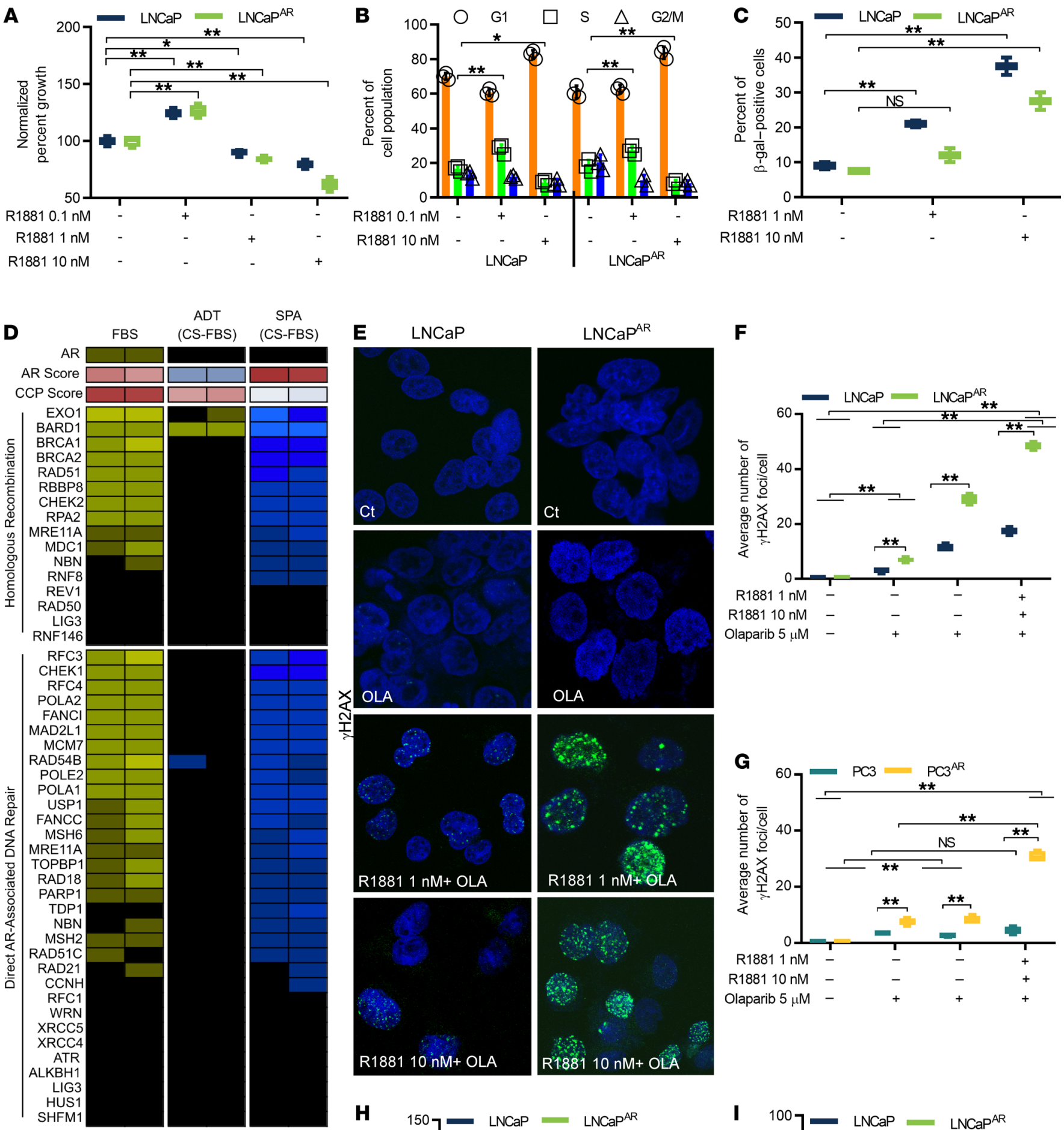

F
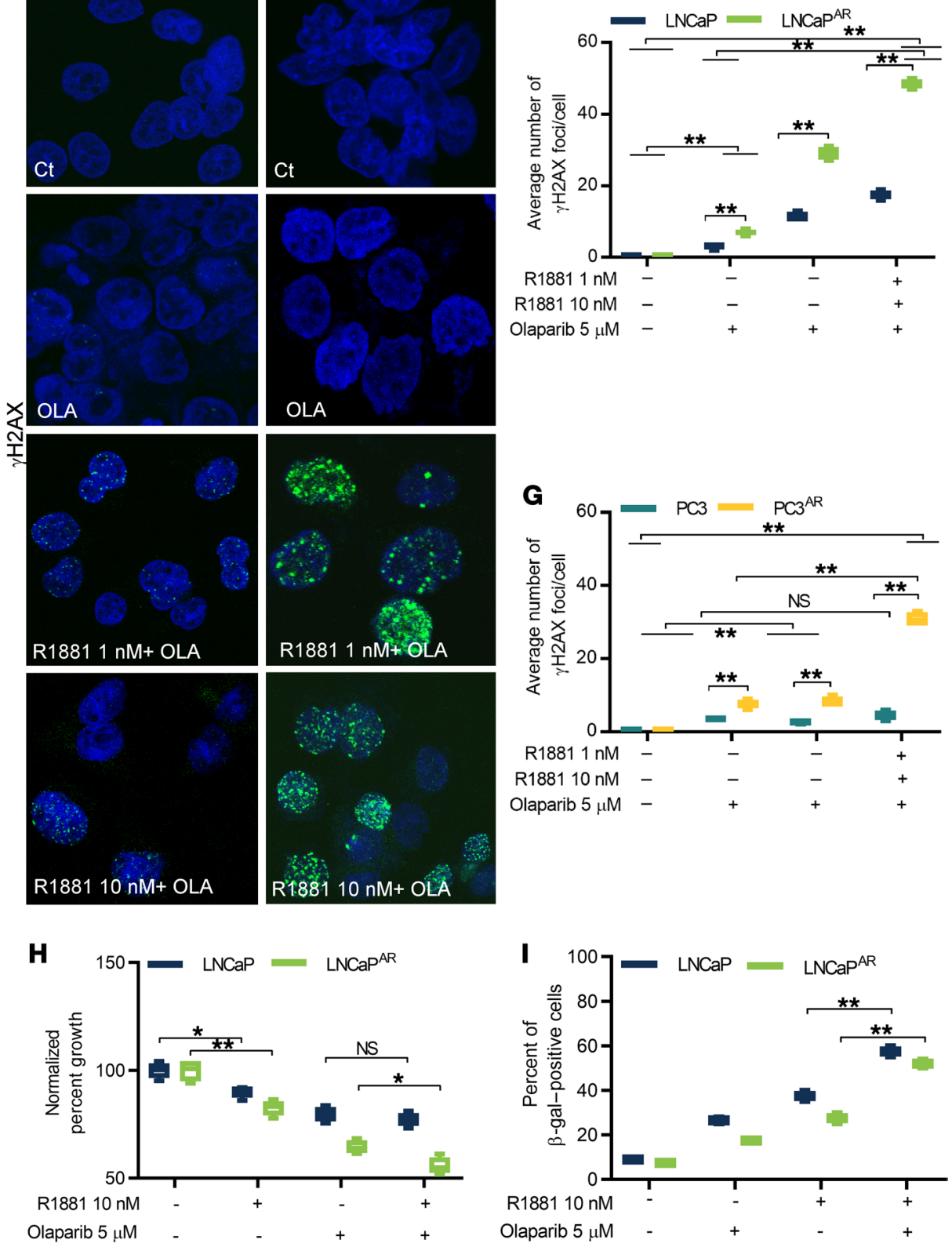
Figure 2. Supraphysiological androgen concentrations influence the growth of PC cells, alter the expression of DNA repair genes, and augment the effects of PARP inhibition. (A) Quantitation of PC cell growth 72 hours after treatment with concentration ranges of R1881. (B) Assessment of cell-cycle phase by flow cytometry 72 hours following treatment with R1881. (C) Assessment of cellular senescence by quantitation of $\beta$-galactosidase staining 72 hours after androgen treatment. (D) Transcript levels determined by RNAseq analysis in LNCaP cells in standard growth medium (FBS) or in androgen-depleted medium, ADT (charcoal-stripped growth medium depleted of androgens), or androgen-depleted medium supplemented with $10 \mathrm{nM}$ R1881, SPA. (E) Confocal immunostaining assay for $\gamma \mathrm{H} 2 \mathrm{AX}$ foci in PC cells in control medium alone (Ct) or supplemented with OLA alone or with R1881. Original magnification: $\times 40$. (F) Quantitation of $\gamma \mathrm{H} 2 \mathrm{AX}$ foci per LNCaP cell. (G) Quantitation of $\gamma \mathrm{H} 2 \mathrm{AX}$ foci per PC3 cell. (H) Quantitation of PC cell growth after treatment with OLA and/or R1881. (I) Assessment of cellular senescence by quantitation of $\beta$-galactosidase staining. In $\mathbf{A}, \mathbf{C}$, and $\mathbf{F}-\mathbf{I}$, data represent the mean $\pm \mathrm{SD}$ ( $n=4$ replicates per experiment). ${ }^{*} P \leq 0.05,{ }^{* *} P<0.01$ by 2 -way ANOVA.

We determined that T exposure, in contrast to DHT and R1881 induced $\gamma \mathrm{H} 2 \mathrm{AX}$ and 53BP1 foci primarily in AR overexpressing $\mathrm{LNCaP}^{\mathrm{AR}}$ and $\mathrm{PC}^{\mathrm{AR}}$ cells and at an earlier 3-hour time point (Supplemental Figure 3, A-F). Previous studies reported that T dissociates from the AR 4 times faster than DHT, and in agreement with these kinetics, by 6 hours after exposure to R1881 or DHT, foci were largely resolved (31).

As DNA damage can occur in the context of cell division with DNA replication, and AR activation can promote cell proliferation, we sought to determine if SPA could induce DNA damage in growth-arrested cells. We used isoleucine-deprived medium to arrest cells in G1 (Supplemental Figure 4A), an approach previously shown not to influence AR expression (32). We observed no increase in DNA damage in $\mathrm{LNCaP}$ or $\mathrm{LNCaP}^{\mathrm{AR}}$ cells grown in isoleucine-deprived medium. The addition of SPA to G1-arrested LNCaP and LNCaPAR cells significantly increased DNA damage as measured by $\gamma \mathrm{H} 2 \mathrm{AX}$ and 53BP1 foci (Supplemental Figure 4, B-E).

$S P A$ increases nuclear $A R$, serine $81 A R$ phosphorylation, and AR transcriptional output. Previous studies have determined that $A R$ occupancy is saturated by ligand concentrations of about $2 \mathrm{nM}\left(K_{D}\right.$ approximately $0.1-2 \mathrm{nM})(33-37)$, and consequently, the mechanisms by which higher pharmacological concentrations of androgens exert biologic effects in PC remain to be established. Notably, the activity of the AR in prostate epithelium is influenced by mass action physiology involving the number of receptors, ligand concentrations, and the affinity and on/off rates of AR ligand interactions. Although AR binding may be saturated in the eugonadal state, the type of androgen, for example T versus DHT, can substantially influence AR activity by altering dissociation rates. Prior studies have demonstrated that higher $\mathrm{T}$ concentrations can overcome more rapid off-rates relative to DHT and overcome the potency deficit by mass action: $\mathrm{T}$ at concentrations above the levels required to saturate AR binding exert effects equivalent to DHT, potentially by stabilizing receptor-hormone complexes (38). Further, high DHT levels and supraphysiological T concentrations (>20 nM) have been shown to increase cellular AR protein levels by enhancing rates of synthesis and reducing turnover (39).

To determine if SPA increases AR nuclear localization, we quantitated AR and serine-81 phosphorylated AR (S81-AR) in PC cells. Phosphorylation of AR at serine- 81 is regulated via $\mathrm{CDK} 1$ and
CDK9 and is vital for ligand-stimulated AR binding to chromatin and for recruiting coactivators needed for transcription (40-42). Compared with PC cells exposed to eugonadal androgen concentrations in standard growth medium, SPA concentrations of $10 \mathrm{nM}$ R1881 or DHT significantly increased nuclear S81-AR levels. PC cells engineered to overexpress AR also exhibited higher nuclear S81-AR, and these levels were further increased by R1881 or DHT (Figure 1, E and F; and Supplemental Figure 5, A-E).

In PC, the liganded AR regulates a diverse gene expression program that includes a subset of repressed genes and a larger number of genes where AR binding activates transcription, including those encoding well-characterized secreted proteins such as PSA/KLK3 (43). We measured transcript levels of KLK3, NKX3.1, and TMPRSS 2 by real-time quantitative reverse transcription PCR (qRT-PCR) 8, 24, and 48 hours following exposure to increasing concentrations of R1881 and DHT. Most notably at 48 hours, each 10-fold increase in R1881 was accompanied by further increases in KLK3 and NKX3.1 and TMPRSS2 transcript abundance (Figure $1 G)$. This association was magnified in $\mathrm{LNCaP}^{\mathrm{AR}}$ cells overexpressing AR (Figure 1G). For DHT, transcript levels peaked at 24 hours and at a lower concentration (10 nM) (Supplemental Figure $5 F)$. Collectively, these results indicate that exposure to SPA can increase AR nuclear localization and AR transcriptional output beyond that observed at eugonadal ligand concentrations reported to saturate AR binding.

SPA inhibits PC cell growth and induces apoptotic and senescence responses in AR-overexpressing PC cells. A consequence of cellular DNA damage is the engagement of potent growth arrest programs that serve to pause the cell cycle to allow repair mechanisms to correct damage or to induce senescence or apoptosis if the extent of damage exceeds repair capacity. To evaluate the effects of SPA on these parameters, we seeded LNCaP, LNCaPAR, PC3, and $\mathrm{PC}^{\mathrm{AR}}$ cells in equal numbers and treated them with androgen levels approximating physiological (0.1-1.0 nM R1881) or SPA (10 nM R1881) concentrations. After 3 days, physiological equivalent androgen levels increased LNCaP cell growth by $24 \%(P<0.01)$, whereas SPA (10 nM R1881) repressed growth by $21 \%(P=0.04)$, and the growth-repressive effects were accentuated in $\mathrm{LNCaP}^{\mathrm{AR}}$ cells expressing high AR levels: $38 \%$ reduction by SPA versus a $26 \%$ increase in growth with $0.1 \mathrm{nM} \mathrm{R} 1881(P<0.01)$ (Figure 2A). The growth of AR-null PC3 and DU145 cells was unaffected by R1881 exposure, whereas SPA repressed the growth of $\mathrm{AR}^{+} \mathrm{VCaP}$ cells by $32 \%$ and $P C 3^{A R}$ cells by $31 \%(P<0.01)$ (Supplemental Figure $6, \mathrm{~A}$ and $\mathrm{B})$. In accordance with these findings, SPA significantly reduced the fraction of $\mathrm{LNCaP}, \mathrm{LNCaP}{ }^{\mathrm{AR}}$, and $\mathrm{PC}^{\mathrm{AR}}$ cells in the $S$ phase of the cell cycle (Figure 2B; Supplemental Figure 6C).

SPA exposure increased apoptosis by $45 \%(P<0.01)$ in PC $3{ }^{\mathrm{AR}}$ cells compared with isogenic WT PC3 cells (Supplemental Figure 6D), although SPA did not increase apoptosis in VCaP, LNCaP, or $\mathrm{LNCaP}^{\mathrm{AR}}$ (Supplemental Figure 6E and data not shown). However, SPA induced a more robust senescence response as measured by the expression of senescence-associated $\beta$-galactosidase (SA- $\beta$ Gal). In physiological androgen concentrations, $5 \%$ of LNCaP cells were SA- $\beta$-Gal positive, whereas $30 \%$ of LNCaP cells stained for SA- $\beta-$ Gal following SPA treatment $(P<0.01)$ (Figure $2 \mathrm{C})$. A significant increase in senescent cells was also measured in $\mathrm{PC} 3^{\mathrm{AR}}$ cells exposed to SPA concentrations (Supplemental Figure 6F). 
A $\quad$ S2056 DNA-PKcs

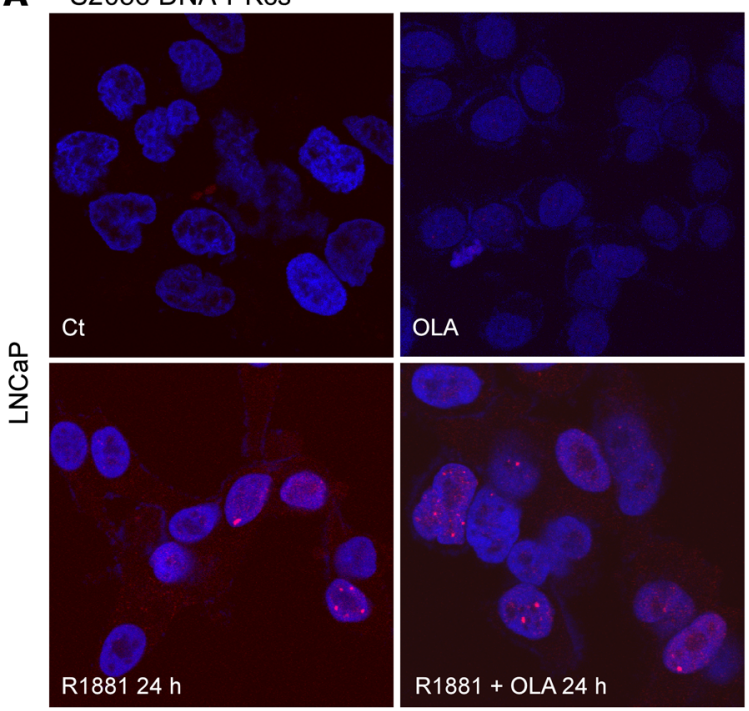

C

LNCaP $=\mathrm{LNCaP}^{\mathrm{AR}}$

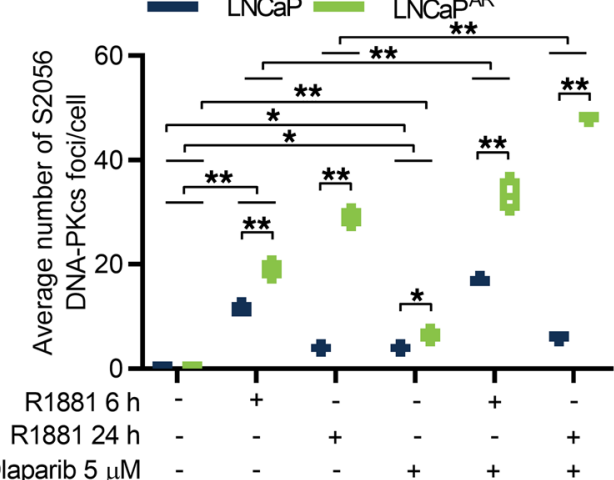

E

T2609 DNA-PKcs

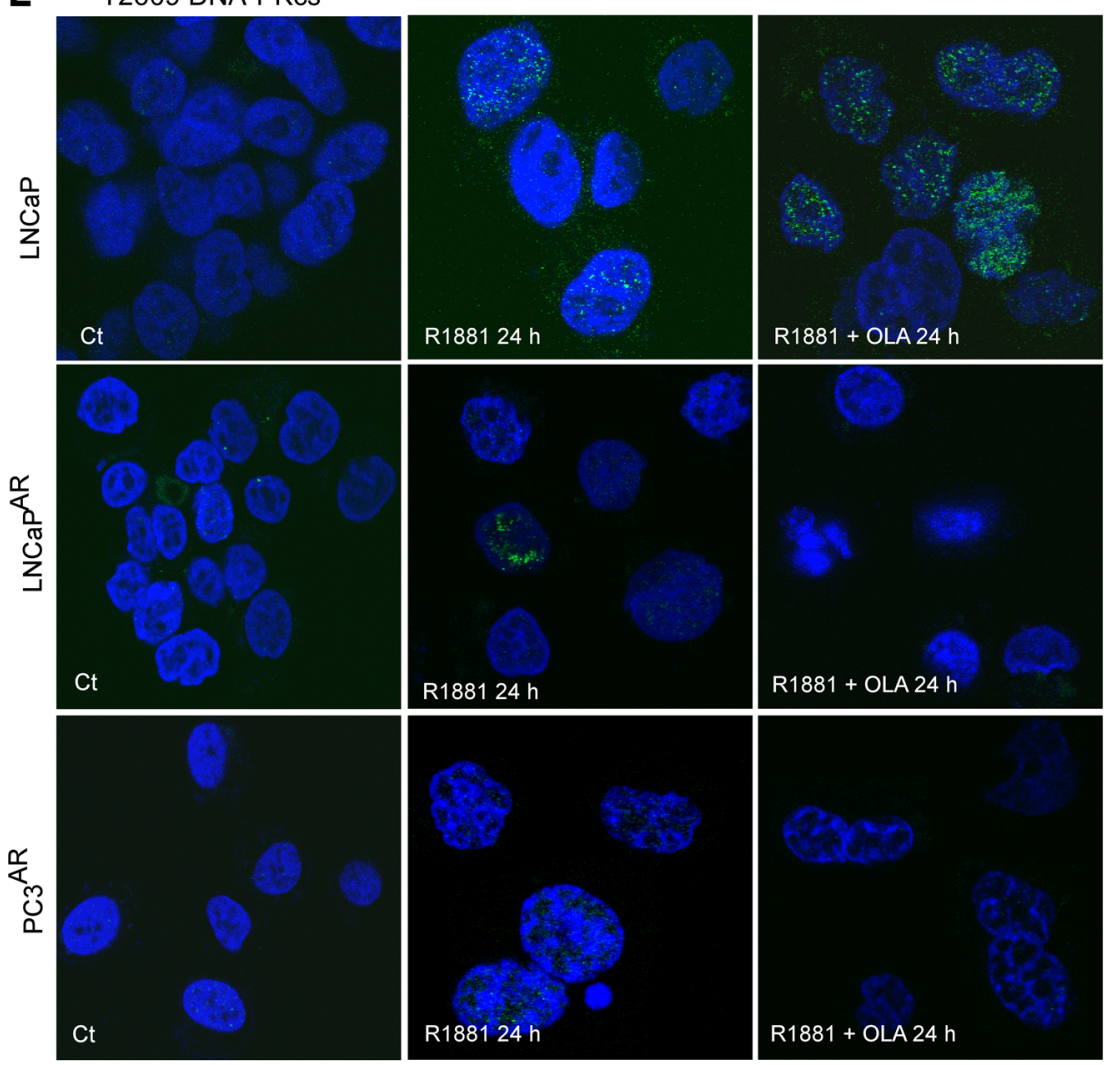

B S2056 DNA-PKcs

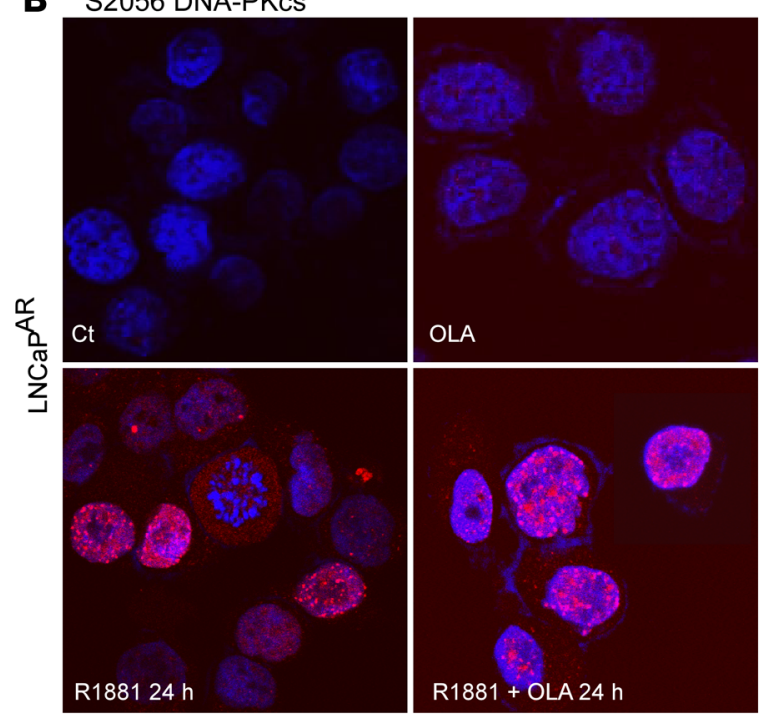

D

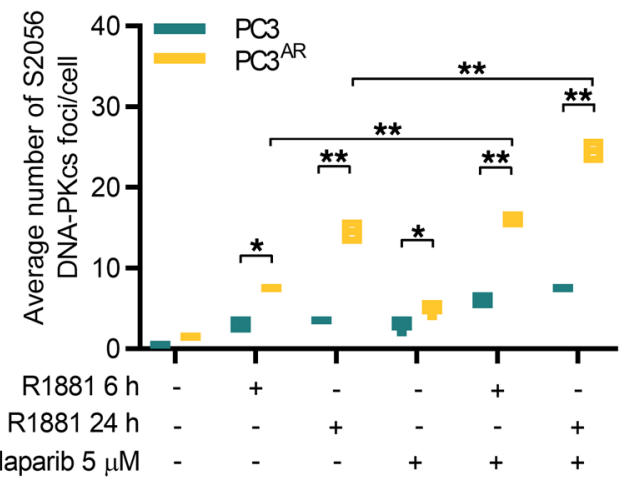

$\mathbf{F}$

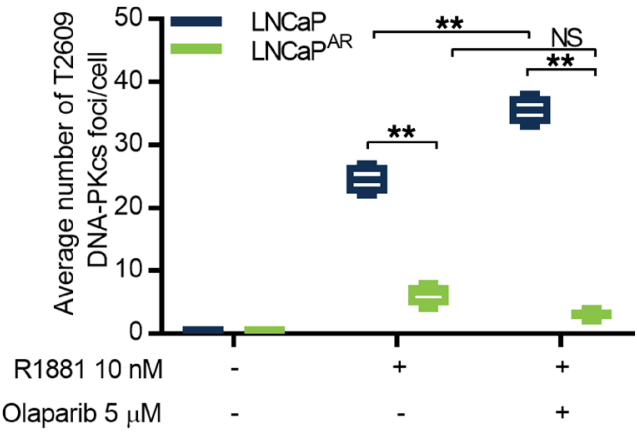

G

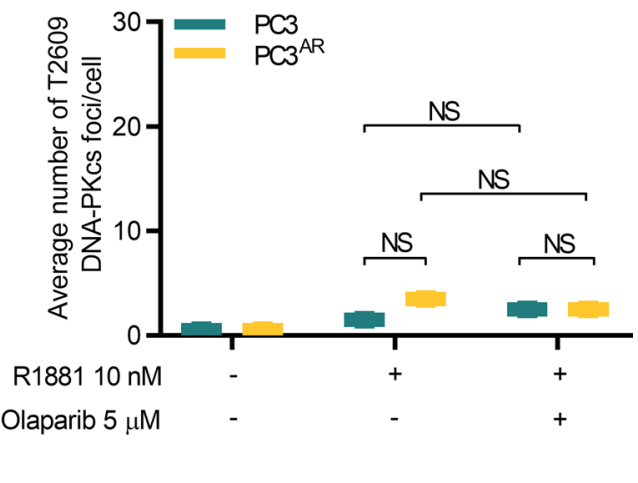


Figure 3. Supraphysiological androgens alter DNA-PKes phosphorylation. Confocal immunostaining of DNA-PKcs S2056 phosphorylation in LNCaP (A) and LNCaPAR (B), 24 hours after treatment with $10 \mathrm{nM} \mathrm{R1881}$ and OLA (C and D). Quantitation of S2056 foci after 6 or 24 hours of R1881 and OLA treatment. (E) Confocal immunostaining of DNA-PKcs T2609 phosphorylation foci in $\mathrm{LNCaP}, \mathrm{LNCaP}{ }^{\mathrm{AR}}$, and $\mathrm{PC} 3^{\mathrm{AR}} 24$ hours after treatment with 10 nM R1881 and/or OLA. (F and G) Quantitation of T2609 foci in cell lines 24 hours after R1881 and OLA treatment. In C, D, F, and $\mathbf{G}$ data represent the mean $\pm \mathrm{SD}(n=4$ replicates per experiment). Original magnification for $\mathbf{A}$ B, and $\mathbf{E}: \times 40 .{ }^{*} P \leq 0.05,{ }^{* *} P<0.01$ by 2 -way ANOVA.

SPA attenuates DNA repair through homology-mediated and nonhomologous end-joining mechanisms. Previous studies have demonstrated that the AR regulates a spectrum of genes involved in repairing DNA damage $(23,24)$. Repressing AR function with ligand depletion or AR antagonists reduces the expression of DNA repair genes and augments the effects of ionizing radiation, including the potentiation of DNA DSBs $(20,23)$. These findings provide a mechanism explaining the clinical benefit of combining ADT with radiotherapy for localized PC $(44,45)$. We confirmed the previous findings by quantitating transcripts encoding genes involved in homology-directed repair (HDR) and nonhomologous end-joining (NHEJ) repair in LNCaP cells exposed to growth medium with charcoal-stripped bovine serum, analogous to ADT. ADT modestly reduced the expression of several genes comprising these pathways (Figure 2D). We also evaluated a panel of DNA repair genes demonstrated to be directly AR regulated by virtue of AR binding to regulatory androgen-response elements (23). ADT modestly reduced the expression of a subset of these genes in LNCaP cells (Figure 2D). Notably, exposure to SPA significantly and substantially reduced the expression of DNA repair genes, most predominantly those involved in HDR and those previously determined to be direct AR targets (Figure 2D). For example, compared with LNCaP cells assessed in normal growth medium, cells assayed in growth medium with charcoal-stripped bovine serum expressed significantly lower levels of BRCA2 transcripts (1.68fold; $P<0.010$ ), whereas exposure to SPA levels (10 nM R1881) reduced BRCA2 transcripts 4 -fold $(P<0.001)$. These results indicate that in addition to inducing DNA damage, SPA represses the genes capable of mediating repair, a finding that may underlie the senescence responses observed in PC cells exposed to high androgen concentrations. We also evaluated the expression of genes involved in other DNA repair pathways such as mismatch repair, base excision repair, and nucleotide excision repair. Most of the changes were not significant and not consistent in LNCaP and VCaP cells, which both exhibit DNA damage following SPA (Supplemental Figure 7, A and B).

To determine if SPA treatment results in a functional impairment of DNA repair, we assessed homologous recombination pathway proficiency by measuring RAD51 foci and the NHEJ pathway activity by DNA end-ligation assays (46). Compared with control, LNCaP and LNCaPAR cells exposed to $10 \mathrm{nM}$ R1881 had no induction of Rad51 foci, indicating compromised HR repair (Supplemental Figure 8A). NHEJ repair was also attenuated by SPA treatment. Nuclear extracts from $\mathrm{LNCaP}, \mathrm{LNCaP}^{\mathrm{AR}}, \mathrm{PC} 3$, and $\mathrm{PC}^{\mathrm{AR}}$ were able to efficiently relegate a linearized plasmid (Supplemental Figure 8B). In contrast, nuclear extracts from LNCaP,
LNCaP ${ }^{\mathrm{AR}}$, and PC3 ${ }^{\mathrm{AR}}$ cells exposed to SPA for 6 or 24 hours failed to ligate DNA. SPA did not affect DNA end ligation in WT AR-null PC3 cells (Supplemental Figure 8B).

PARP inhibition augments SPA-induced DNA damage and induces cellular senescence. Having established that androgens consistently induce DNA damage in PC cells through mechanisms that are sensitive to both AR levels and ligand concentrations, we next sought to determine if inhibiting DNA repair mechanisms would further augment SPA-induced DNA damage and promote apoptosis or senescence. We first targeted PARP1, a multifunctional enzyme involved in the repair of DNA strand breaks (47). Treatment with the PARP1 inhibitor olaparib (OLA) slightly increased the number of $\gamma \mathrm{H} 2 \mathrm{AX}$ foci from approximately $1 \mathrm{FPC}$ in vehicle-treated LNCaP cells to 4 FPC in OLA-treated cells $(P<$ 0.01) (Figure 2, E and F). A similar response was observed in PC3 cells (Figure 2G). Overexpression of the AR in both lines increased OLA-induced DSBs $(P<0.001)$. Further, OLA exposure substantially increased both $\gamma \mathrm{H} 2 \mathrm{AX}$ and 53BP1 foci in LNCaP, LNCa$\mathrm{P}^{\mathrm{AR}}$, and PC3 ${ }^{\mathrm{AR}}$ cells when given concurrently with SPA, and the extent of DSBs was associated with AR expression. The number of $\gamma \mathrm{H} 2 \mathrm{AX}$ FPC increased from a baseline of 1 in vehicle-treated LNCaP to 16 in LNCaP cells treated with both OLA and $10 \mathrm{nM}$ R1881 $(P<0.01)$ to $49 \mathrm{FPC}$ in $\mathrm{LNCaP}^{\mathrm{AR}}$ cells treated with both OLA and $10 \mathrm{nM}$ R1881 $(P<0.01)$ (Figure 2, E and F). OLA treatment alone modestly reduced the growth of $\mathrm{LNCaP}$ or $\mathrm{LNCaP}^{\mathrm{AR}}$ cells by $10 \%$ and $17.5 \%$, respectively (Figure $2 \mathrm{H}$ ). The addition of OLA to SPA further augmented the growth-repressive effects of SPA alone, from $38 \%-45 \%$ in LNCaPAR cells, although OLA did not further augment effects of SPA on $\mathrm{PC}^{\mathrm{AR}}$ growth (Figure $2 \mathrm{H}$; Supplemental Figure $6 \mathrm{H})$. The combination of OLA and SPA did not increase apoptosis rates (Supplemental Figure 6D), but the number of senescent cells was substantially increased. The addition of OLA to SPA increased SA- $\beta$-Gal-positive cells from $30 \%-50 \%$ $(P<0.01)$ and $20 \%-35 \%(P<0.01)$ in $\mathrm{LNCaP}^{\mathrm{AR}}$ and $\mathrm{PC} 3^{\mathrm{AR}}$ cells, respectively (Figure 2I; Supplemental Figure 6G).

DNA-PKcs inhibition attenuates SPA-induced DNA damage and promotes cell survival. The mechanisms promoting the repair of DNA DSBs exhibit cell-cycle dependency with homology-directed repair occurring in S/G2 and NHEJ occurring throughout the cell-cycle phases where it is the predominant mechanism of repair in G1 (48). As previously reported, SPA induces PC cell growth arrest in G1 (Figure 2B) $(49,50)$; therefore, instead of HR, AR/ SPA-induced DSBs should primarily undergo repair by NHEJ. To evaluate NHEJ activity, we focused on a key regulatory component of NHEJ, DNA-dependent protein kinase, catalytic subunit, DNA-PKcs. Following DNA DSBs, DNA-PKcs is recruited by the DNA end-binding Ku70/80 heterodimer and undergoes rapid autophosphorylation at S2056 (pS2056) $(46,51,52)$. Subsequent phosphorylation on T2609 (pT2609; in the ABCDE cluster) by ATM or ATR leads to its dissociation from the DNA ends, which is required for the progression of NHEJ repair (52-54).

Treatment of LNCaP cells with $10 \mathrm{nM}$ R1881 produced significant increases in pS2056 DNA-PKcs foci after 6 hours (from 1-11 FPC; $P=0.05$ ) (Figure 3, A and C), indicating hyperactivation of DNA-PKcs, followed by foci resolution after 24 hours (Figure 3C). $\mathrm{LNCaP}^{\mathrm{AR}}$ and $\mathrm{PC} 3^{\mathrm{AR}}$ cells showed augmented and sustained pS2056 DNA-PKcs foci. At 6 hours, the number of FPC in LNCaP 
A $\gamma \mathrm{H} 2 \mathrm{AX}$
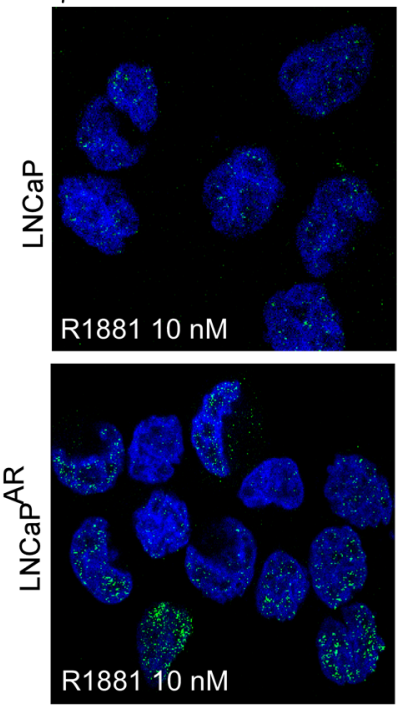

$\mathrm{R} 1881+\mathrm{Nu} 7441$

\section{S2056 DNA-PKcs}
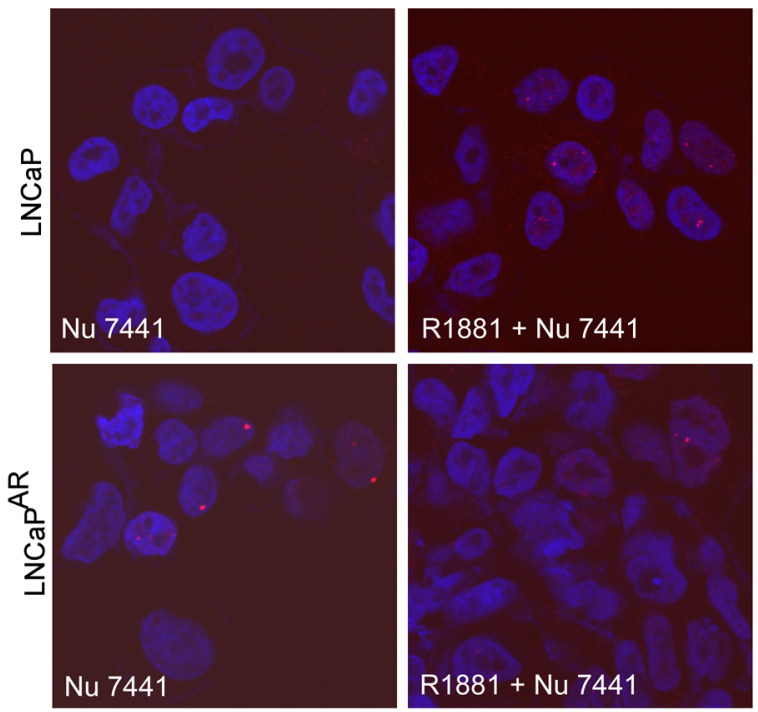

E

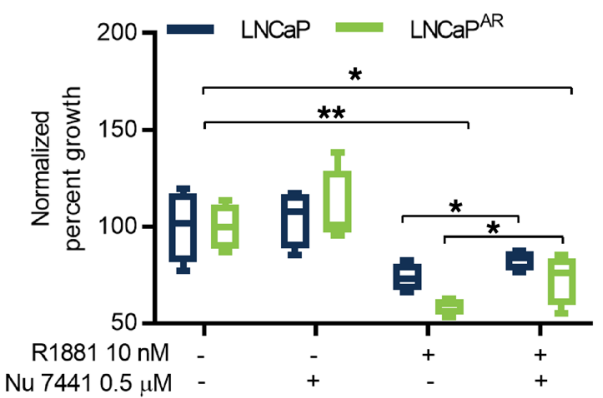

B
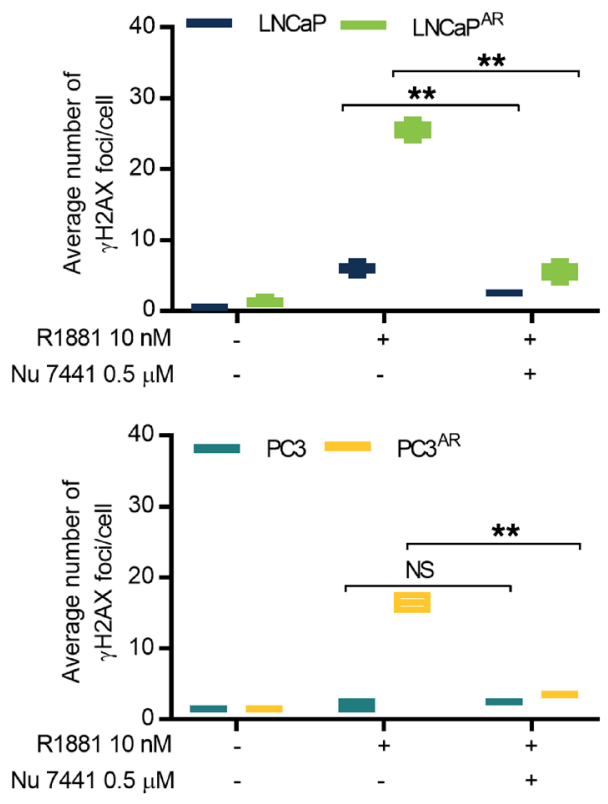

D S81 AR

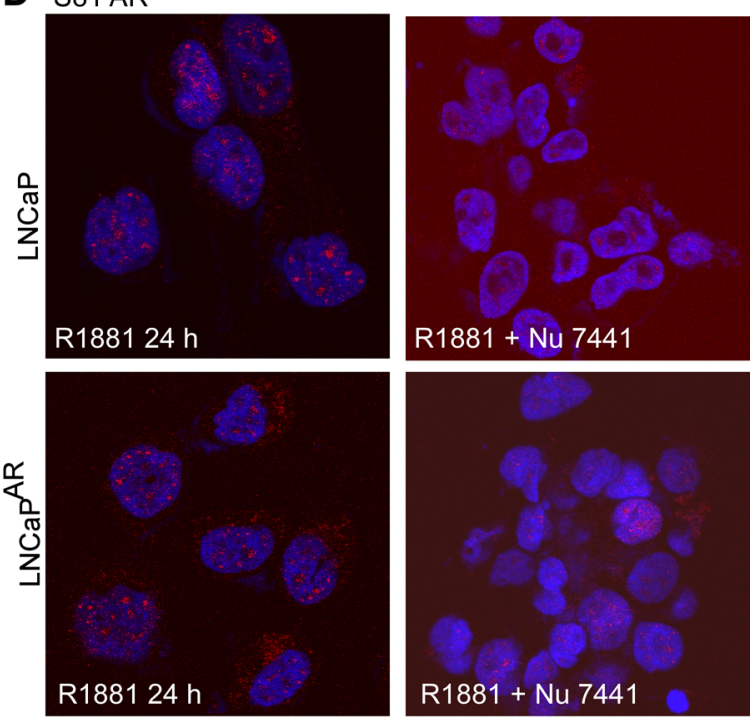

$\mathbf{F}$

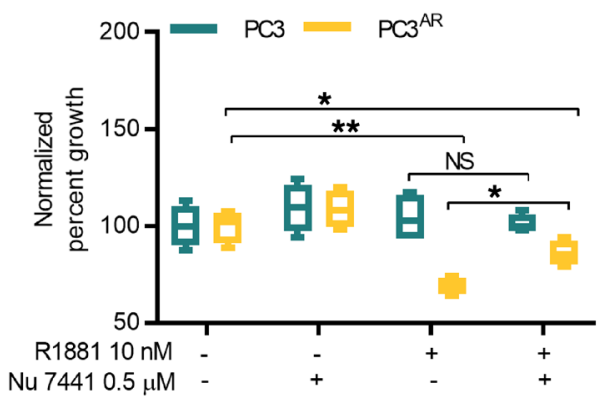

Figure 4. Inhibition of DNA-PKcs attenuates SPA-induced DNA damage and PC growth repression. (A) Confocal immunostaining of $\gamma \mathrm{H} 2 \mathrm{AX}$ foci LNCaP and LNCaPAR cells. (B) Quantitation of $\gamma \mathrm{H} 2 \mathrm{AX}$ foci LNCaP and LNCaPAR cells (top) and PC3 and PC ${ }^{A R}$ cells (bottom) with DNA-PKcs inhibition and exposure to supraphysiological androgens. (C) Confocal immunostaining of DNA-PKcs S2056 foci and (D) AR S81 foci, respectively in LNCaP and LNCaPAR cells after 24-hour treatment with SPA with 1-hour pretreatment with DNA-PKcs inhibitor Nu7441. (E and F) Quantitation of LNCaP, LNCaPAR, and PC3 and PC3 ${ }^{A R}$ cell growth following 3 days of treatment with the DNA-PKcs inhibitor Nu7441 and/or $10 \mathrm{nM}$ R1881. In B, E, and F, data represent the mean \pm SD $(n=4$ replicates per experiment). Original magnification for $\mathbf{A}, \mathbf{C}$, and $\mathbf{D}: \times 40 .{ }^{*} P \leq 0.05,{ }^{*} P<0.01$ by 2 -way ANOVA. 
and LNCaPAR cells measured 10 and 19 , respectively $(P<0.01)$ and at 24 hours, the number of FPC in LNCaP and $\mathrm{LNCaP}^{\mathrm{AR}}$ cells measured 4 and 29, respectively $(P<0.01)$ (Figure 3, B and $C$ ). As S2056 phosphorylation is dose dependent, increased DNA damage will induce more pS2056 foci, which is concordant with more damage foci observed in $\mathrm{LNCaP}^{\mathrm{AR}}$ and $\mathrm{PC}^{\mathrm{AR}}$ cells compared with the parental cells. $\mathrm{AR}^{+} \mathrm{VCaP}$ cells also demonstrated pS2056 DNA-PKcs foci following SPA treatment, whereas in AR-null PC3 and LNCaPAIPC cells, no pS2056 DNA-PKcs foci were observed (Figure 3D; Supplemental Figure 8, C and D).

We next sought to determine the effects of PARP inhibition with respect to DNA-PKcs activity following SPA-induced DNA damage. OLA treatment alone had insignificant effects on either S2056 or T2609 DNA-PKcs phosphorylation (Figure 3, A-D), consistent with the minimal induction of DNA damage resulting from single-agent OLA (Figure 2, $\mathrm{E}-\mathrm{G}$ ). In contrast, the addition of OLA to SPA significantly increased pS2506 foci in AR-expressing PC cells (Figure 3, B-D). These foci peaked at 6 hours and largely resolved in parental LNCaP cells 24 hours after treatment, but further increased in the $\mathrm{LNCaP}^{\mathrm{AR}}$ and $\mathrm{PC} 3^{\mathrm{AR}}$ cells expressing high levels of AR (Figure 3, C and D). Collectively, these findings may reflect the PARP1 trapping effects of OLA that delay PARP dissociation and impair DNA repair (55).

Previous reports determined direct interactions between AR and DNA-PKcs, with DNA-PKcs serving as a coactivator of AR transcriptional function (24). Regulated transcription by nuclear hormone receptors involve DNA DSBs that are mediated by TOP2B and a complex that includes PARP1, Ku70/80, and DNAPKcs $(21,22)$. We hypothesized that AR over-expression and enhancement of AR activity via SPA, may serve to maintain this regulatory complex on DNA and impair dissociation. In order to determine the status of DNA-PKcs dissociation, we examined T2609 phosphorylation, which is a necessary event for subsequent DNA-PKcs displacement required for the remaining steps in NHEJ repair (54). Parental LNCaP cells treated with SPA exhibited the expected autophosphorylation event at S2056 followed by the resolution of these foci at 24 hours and the gain of pT2609 foci at 24 hours (Figure 3, C, E, and F). SPA further increased pS2056 foci (24 hours) in $\mathrm{LNCaP}^{\mathrm{AR}}$ cells overexpressing AR without measurable changes in pT2609 foci (Figure 3, E and F), indicating that DNA-PKcs is hyperactivated and persists on chromatin in AR overexpressing cells leading to defective dissociation and potentially impaired DNA repair (Supplemental Figure 8E). We also did not observe substantial changes in pT2609 foci in PC ${ }^{\mathrm{AR}}$ cells exposed to SPA (Figure 3G). OLA treatment increased pT2609 DNA-PKcs foci in $\mathrm{LNCaP}$ cells but not in $\mathrm{LNCaP}^{\mathrm{AR}}$ or PC ${ }^{\mathrm{AR}}$ cells where these foci were not observed (Figure 3, F and G).

We next sought to determine whether inhibition of DNAPKcs would reduce or augment DNA damage induced by SPA. Whereas SPA exposure induced DNA DSBs in LNCaP cells, with further increases in AR over-expressing $\mathrm{LNCaP}^{\mathrm{AR}}$ and $\mathrm{PC}^{\mathrm{AR}}$ cells, treatment with the DNA-PKcs inhibitor Nu7441 eliminated the induction of $\gamma \mathrm{H} 2 \mathrm{AX}$ foci (Figure 4, A and B). Treatment of LNCaP and LNCaPAR cells with Nu7441 nearly abolished the DNA-PKcs pS2056 foci resulting from SPA exposure (Figure 4C). Treatment with Nu7441 also substantially reduced nuclear S81-AR foci and S81-AR phosphorylation, supporting previous studies demonstrat- ing a coregulatory role for DNA-PKcs enhancing AR activity (Figure 4D, Supplemental Figure 8F) (24). The abrogation of measurable DNA damage by DNA-PKcs inhibition translated to the loss of PC growth repression without the induction of apoptosis and senescence following SPA treatment. Individually, SPA (but not $\mathrm{Nu} 7441$ ) reduced cell viability by $21 \%$ relative to vehicle in $\mathrm{LNCaP}$ cells. However, the addition of Nu7441 to SPA enhanced the growth of $\mathrm{LNCaP}, \mathrm{LNCaP}{ }^{\mathrm{AR}}$, and $\mathrm{PC} 3^{\mathrm{AR}}$ cells relative to SPA alone (Figure 4, E and F). The growth of AR-null PC3 cells was not affected by either single agent or combination drug treatment (Figure $4 \mathrm{~F}$ ).

PCs deficient in BRCA2 exhibit enhanced sensitivity to SPA, PARP antagonists, and DNA-PKcs inhibition. Recent molecular profiling studies have determined that approximately $20 \%$ of metastatic PCs exhibit aberrations in genes mediating HDR, most notably BRCA2 (56). In HDR-defective cells, most of the DNA damage repair occurs by NHEJ. We hypothesized that in the absence of HDR, SPA treatment would induce more substantial DNA damage and consequent impairment of cell survival. To evaluate this possibility, we created a tetracycline-inducible shRNA BRCA2 cell line ( $\mathrm{LNCaP}^{\mathrm{shBRCA} 2}$ ) and separately genetically modified LNCaP cells by CRISPR/Cas9 to generate a line of BRCA2-deficient LNCaP cells (LNCaP ${ }^{\mathrm{BRCA}}$ ) (Figure 5A and Supplemental Figure 9A). Compared with parental LNCaP, SPA induced substantially greater $\gamma \mathrm{H} 2 \mathrm{AX}$ foci in $\mathrm{LNCaP}^{\text {shBRCA2}}$, at $5 \mathrm{FPC}$ versus $33 \mathrm{FPC}(P<0.001)$ (Figure $5, \mathrm{~B}$ and $\mathrm{C}$ ). Similar effects were observed in $\mathrm{LNCaP}^{\mathrm{BRCA} 2}$ cells (Supplemental Figure 9, B and D). The enhanced effects of SPA in LNCaPshBRCA2 were further augmented by OLA with $33 \gamma \mathrm{H} 2 \mathrm{AX}$ foci produced by SPA, 23 foci by OLA, and 50 foci produced by SPA plus OLA (Figure 5, B and C). Similar effects were observed in LNCaP ${ }^{\mathrm{BRCA} 2}$ cells exposed to SPA and OLA (Supplemental Figure 9, B and D). SPA also induced a greater number of pS2056 DNA PKcs foci, which was further increased by OLA (Figure 5, D and E, and Supplemental Figure 9, C and E). Treatment of LNCaPshBRCA2 cells with SPA, the DNA-PKcs inhibitor Nu7441, or OLA increased caspase activity by $15 \%(P<0.01), 22 \%(P<0.01)$, and $13 \%(P<$ 0.05 ), respectively (Figure $5 \mathrm{~F}$ ), with similar effects observed in the LNCaP ${ }^{\text {BRCA2 }}$ cells (Supplemental Figure 9F). Combining Nu7441 with SPA further augmented caspase activity and growth repressive effects of SPA: 15\% growth inhibition with SPA alone versus $37 \%$ with the combination of SPA and Nu7441 $(P<0.01)$ (Figure 5G, Supplemental Figure 9G). Exposure of LNCaP cells to doxycycline did not enhance DNA damage, trigger caspase activity, or reduce growth significantly (Supplemental Figure 9, H-J).

To provide further evidence supporting the hypothesis that HR-deficient PCs exhibit enhanced sensitivity to SPA-induced DNA damage, we evaluated the acute in vitro effects of SPA in 3 patient-derived xenograft (PDX) lines: LuCaP96CR with BRCA2 loss and LuCaP35 and LuCaP70, without known HDR defects (Supplemental Figure 10, A and B) (57). Cells from PDX tumors were established as short-term in vitro cultures, and effects following acute exposure to SPA (18 hours) or longer treatment (72 hours) were measured either by DNA damage markers or by growth and caspase assays. At a time point 18 hours after SPA treatment, LuCaP96CR cultures exposed to $10 \mathrm{nM}$ R1881 increased $\gamma \mathrm{H} 2 \mathrm{AX}$ foci from a steady-state level of 3-36 FPC $(P<0.01)$ (Figure 5, $\mathrm{H}$ and I). OLA treatment also increased $\gamma \mathrm{H} 2 \mathrm{AX}$ foci from 2-3 FPC to 20 FPC and the combination of SPA and OLA increased 
A

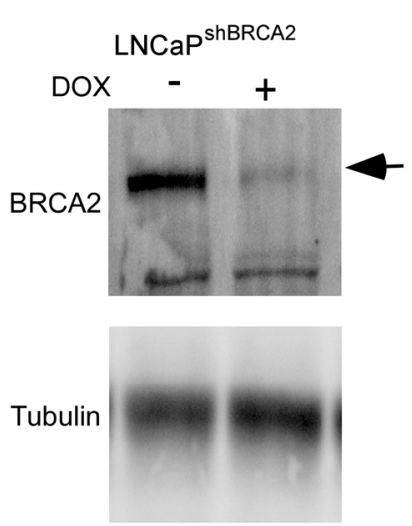

C

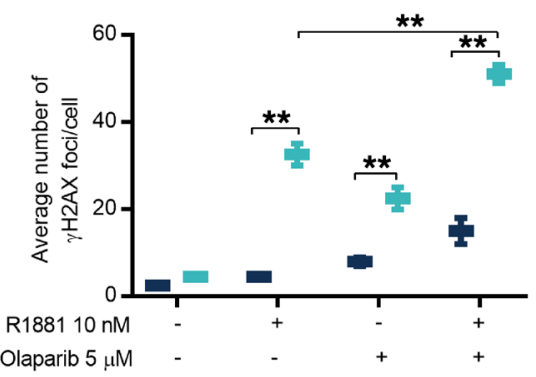

Olaparib $5 \mu \mathrm{M}$
B

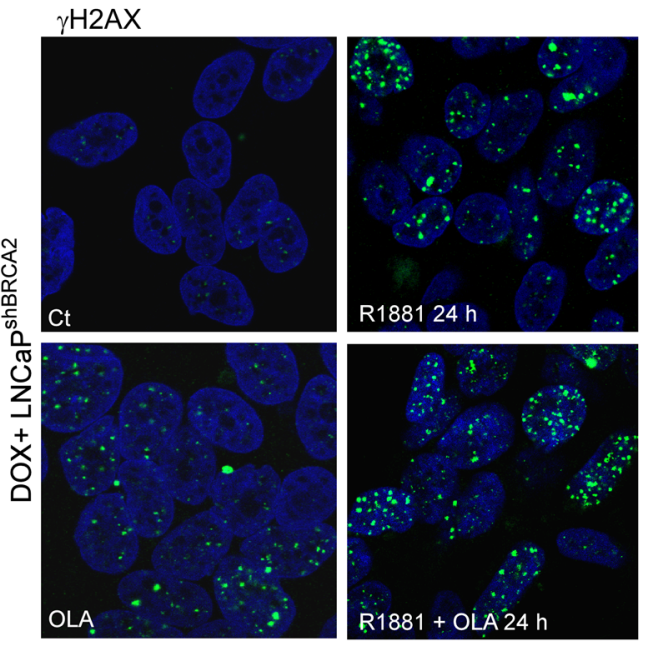

D S2056 DNA-PKcs
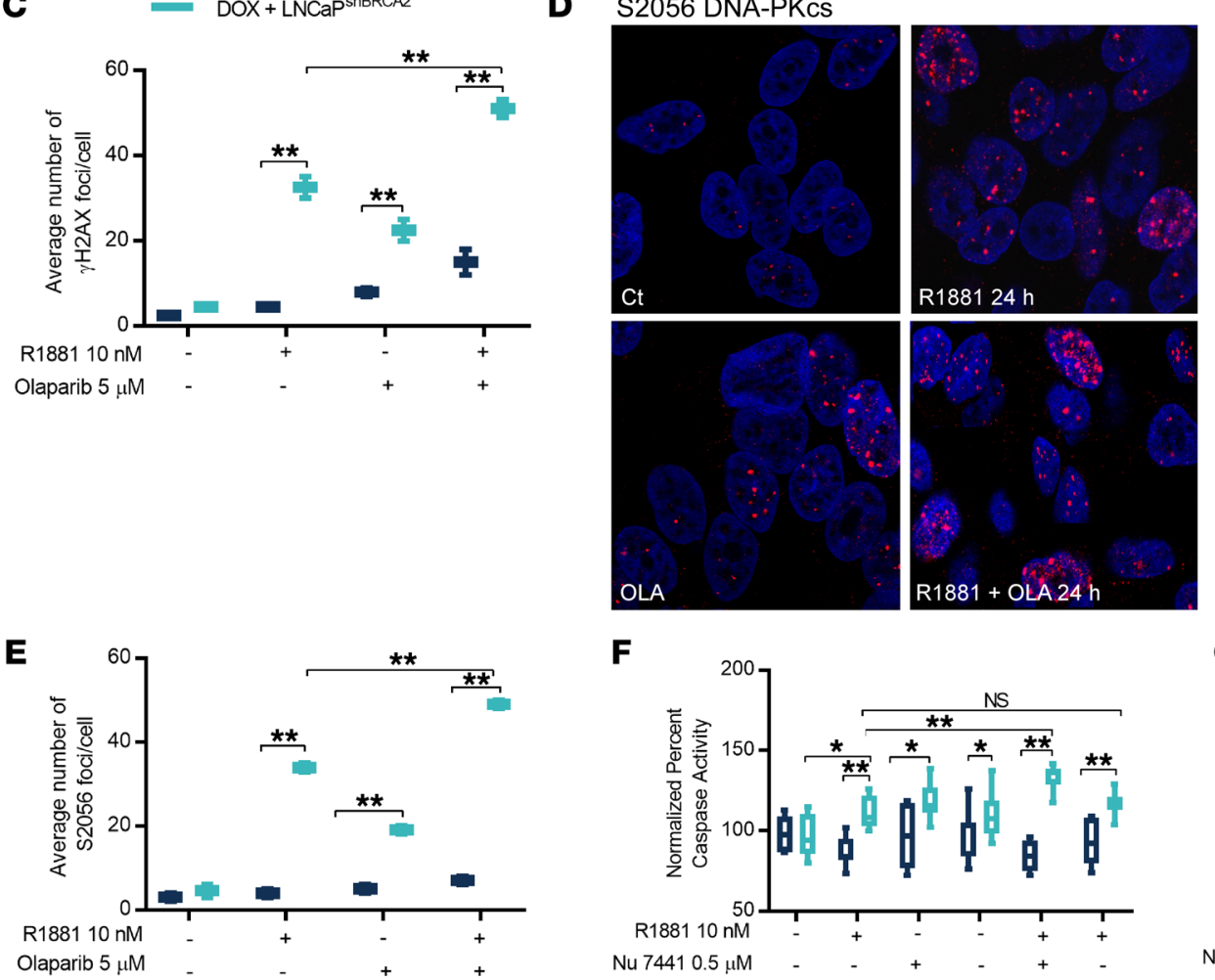

$\mathbf{F}$

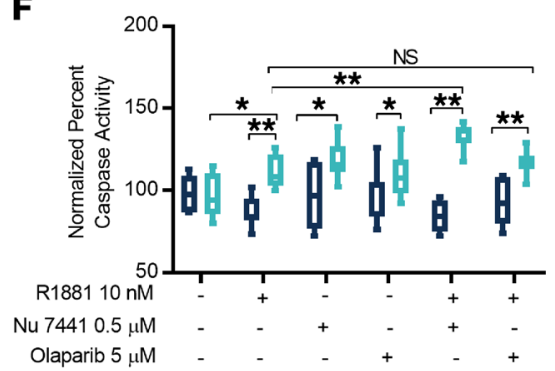

Figure 5. SPA-induced DNA damage and repression of $P C$ growth are enhanced by BRCA2 loss and PARP inhibition.

(A) Western immunoblot of BRCA2 in protein extracts from $\mathrm{LNCaP}{ }^{\text {shBRCA2 }}$ cells in the presence or absence of doxycycline (DOX). (B) Confocal immunostaining and (C) quantitation of $\gamma \mathrm{H} 2 \mathrm{AX}$ in $\mathrm{LNCaP}{ }^{\text {shBRCA2 }}$ cells following $10 \mathrm{nM}$ R1881 and/or OLA treatment for 24 hours in the presence of DOX. (D) Confocal immunostaining and (E) quantitation of DNA PKcs S2056 foci in DOX-treated LNCaPshBRCA2 cells following 10 nM R1881 and/or OLA treatment for 24 hours. (F) Quantitation of apoptosis by caspase activity and (C) growth of $L N C a P^{\text {shBRCA2 }}$ cells in the presence or absence of DOX after 3 days treatment with R1881, Nu7441, or OLA. (H) Confocal immunostaining of $\gamma \mathrm{H} 2 \mathrm{AX}$ and DNA-PKcs S2056 foci in dissociated cells from the PC BRCA2 $2^{-1-}$ LuCaP96 PDX line, exogenously treated with $10 \mathrm{nM}$ R1881 with or without OLA for 18 hours. (I) Quantitation of $\gamma \mathrm{H} 2 \mathrm{AX}$ foci in dissociated cells from HR-intact LuCaP35, LuCaP70, and HR-deficient LuCaP96CR cells following 4 and 18 hours of R1881 or OLA treatment. In C, E, F, G, and I, data represent mean $\pm S D$ ( $n=4$ replicates per experiment). Original magnification for B, D, and $\mathbf{H}: \times 40 .{ }^{*} P \leq 0.05,{ }^{* *} P<0.01$ by 2-way ANOVA.
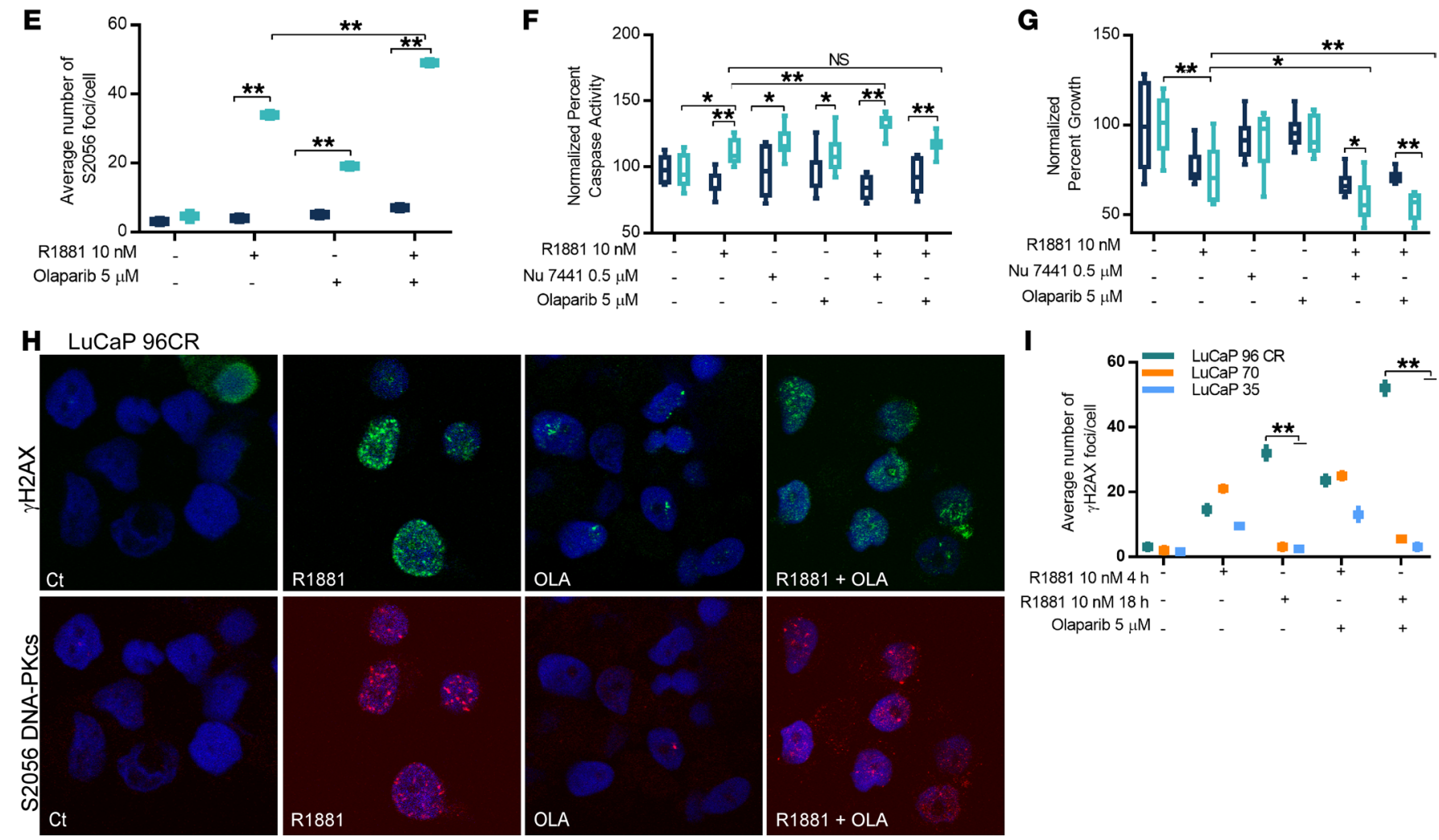

I

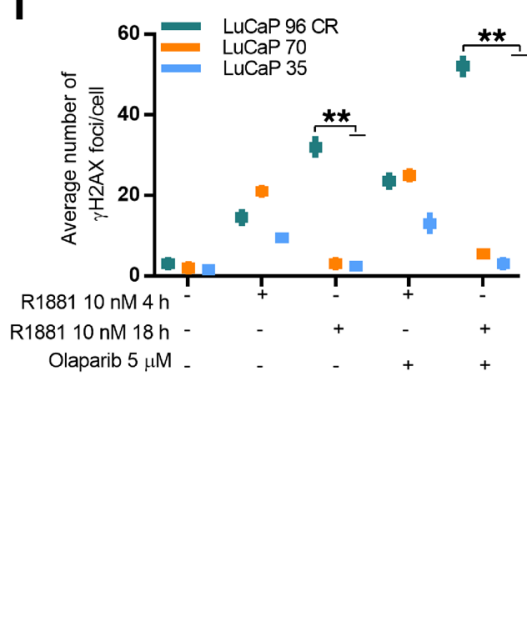


Homologous recombination gene mutation

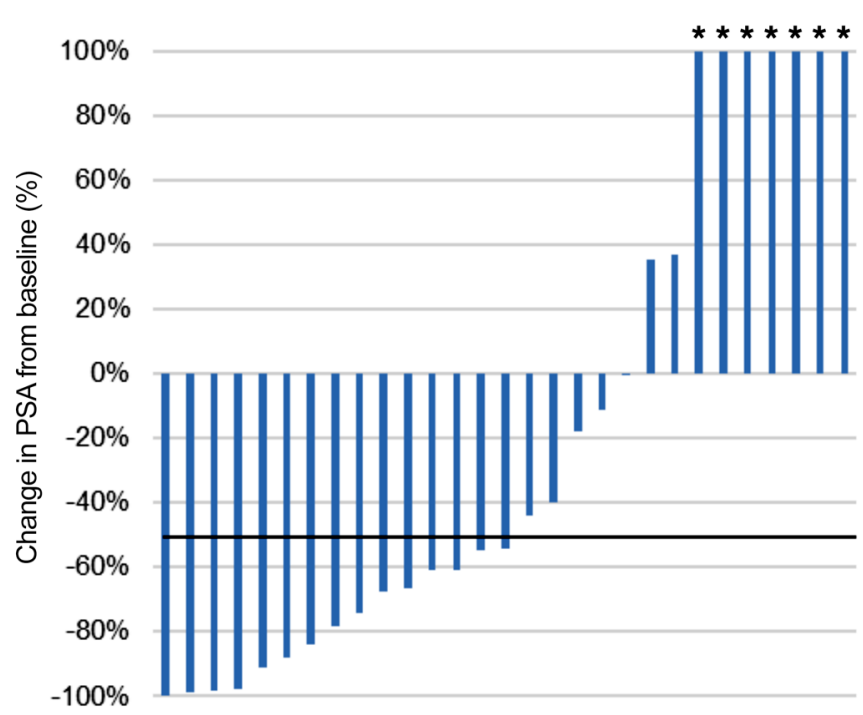

No homologous recombination gene mutation

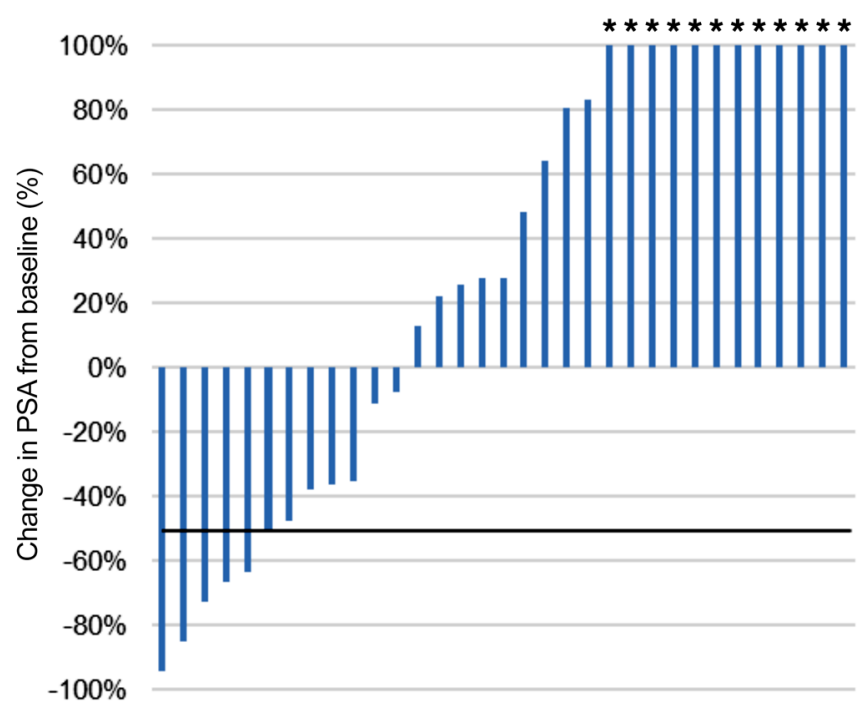

Figure 6. Clinical response to supraphysiological T treatment is associated with mutations in homologous recombination DNA repair genes. PSA waterfall plot for patients receiving BAT as part of 2 ongoing phase II trials. Data are presented for patients with and without pathogenic germline or somatic mutations in HR DNA repair pathway genes (i.e., HRD). PSA declines of greater than or equal to $50 \%$ ( $\mathrm{PSA}_{50}$ response) were more frequent in patients with HRD compared with those without HRD (PSA ${ }_{50}$ response: $15 / 29$ [52\%] vs. $6 / 33[18 \%] ; \chi^{2} P=0.005$ ). *Percent change in PSA truncated at $100 \%$.

the foci number to 50 FPC $(P<0.01)$ (Figure $5, \mathrm{H}$ and I). SPA also increased DNA-PKcs pS2056 foci from 3 FPC in vehicle-treated cells to 34 FPC in cells treated with SPA, and the combination of SPA and OLA further increased pS2056 FPC from 30 to $45(P<$ 0.01) (Supplemental Figure 10C).

In contrast to the marked DNA damage effects observed in LuCaP96CR cells, tumor cells from LuCaP35 and LuCaP70 PDX lines exhibited modest increases in $\gamma \mathrm{H} 2 \mathrm{AX}$ FPC following SPA treatment (from 2 to 11 and from 2 to 22, respectively) measured 4 hours after SPA treatment or the combination of SPA and OLA (Figure 5I). The damage was largely resolved in these cells by 18 hours, whereas significantly greater damage foci persisted in LuCaP96CR cells (Figure 5I).

We confirmed these findings ex vivo using tissue-slice cultures of castration-resistant LuCaP35CR and LuCaP96 tumors to maintain the 3D architecture of tumor cells and microenvironment constituents. After 3 days of exposure to SPA, LuCaP96 tumors exhibited persistent DNA damage in the form of $\gamma \mathrm{H} 2 \mathrm{AX}$ foci (Supplemental Figure 10, D and E). The combination of OLA and SPA further induced damage foci $(P<0.01)$ compared with SPA or OLA alone. Although acute exposure to SPA and/or OLA increased $\gamma \mathrm{H} 2 \mathrm{AX}$ foci in LuCaP35CR cells, which have intact BRCA2, the foci did not persist and were equivalent to pretreatment levels 3 days after treatment (Supplemental Figure 10, D and E).

Patients with PC who have DNA repair deficiency exhibit enhanced clinical responses to SPA. Given that SPA is able to induce DNA damage in PC preclinical models, we hypothesized that mutations in genes involved in DNA damage repair would associate with improved responses to bipolar androgen therapy (BAT) in patients with CRPC (10). To test this, we obtained biospecimens from patients with CRPC enrolled in ongoing clinical trials testing $\mathrm{BAT}$, a form of intermittent high-dose $\mathrm{T}$ designed to produce rapid fluctuations in serum $\mathrm{T}$ from the near-castrate to supraphysiological (> $1500 \mathrm{ng} / \mathrm{dL}$ ) range over the course of 1 month (10). We performed germline or somatic next-generation sequencing (NGS) on biospecimens that included plasma (i.e., cell-free DNA) $(n=$ $79)$, tumor tissue $(n=21)$, and saliva $(n=10)$. Several clinical-grade NGS platforms were used. Given the concern for false negatives, cases were excluded if plasma NGS did not reveal any somatic alteration. Absence of a germline alteration was not assumed to indicate absence of somatic alterations.

Most patients received BAT following 1 or more next-generation AR pathway antagonists, while 6 received BAT as first-line CRPC therapy. Of 65 cases where a germline or somatic pathogenic alteration in any gene was detected, 29 (45\%) had evidence of homology-directed repair deficiency (HRD), with mutations found in BRCA2 $(n=10), A T M(n=8), C H E K 2(n=5)$, PALB2 $(n=$ 3), CDK12 $(n=3)$, CHD1 $(n=2)$, FANCA $(n=1)$, FANCD2 $(n=1)$, and BRCA1 $(n=1)$. Mutations in HR genes associated with increased PSA $_{50}$ responses (i.e., $\geq 50 \%$ decline in PSA from baseline): 15/29 (52\%) patients with HRD demonstrated a PSA $_{50}$ response compared with only 6/33 (18\%) patients without HDR $\left(\chi^{2} P=0.005\right)$ (Figure 6, Supplemental Table 1).

\section{Discussion}

Despite compelling preclinical evidence demonstrating that exposure to high concentrations of androgens can retard PC growth, particularly after adaptation to ADT, clinical outcomes have not been consistent. Four small contemporary trials of $\mathrm{T}$ treatment in CRPC have been reported, with 2 trials of continuous administration that did not achieve supraphysiological levels showing limited clinical responses $(58,59)$ and 2 trials using an intermittent "bipolar" treatment regimen achieving transient supraphysiological levels reporting more robust responses with PSA declines (> 50\%; 
$\left.\mathrm{PSA}_{50}\right)$ or radiographic responses in $30 \%-50 \%$ of men $(10,11)$. Our results indicate that high SPA concentrations combined with high AR levels produce the most substantial and sustained DNA damage, with attendant growth arrest and cellular senescence. These results may explain divergent clinical responses observed in patients treated with different $\mathrm{T}$ regimens.

The AR positively regulates a spectrum of DNA repair genes, and repressing AR activity is documented to attenuate DNA repair and promote radiation-induced cytotoxicity $(23,24)$. However, prior studies have determined that the expression of several DNA repair genes (e.g., BRCA2, ATM, and others) in CRPC is inversely related to AR activity (60) and the AR has been shown to directly repress genes involved in DNA replication including several with repair functions (25). We confirmed that several genes encoding DNA damage/ repair proteins are downregulated by ADT in vitro, notably those involved in homology-directed repair, but we also determined that SPA exposure repressed these genes to a significantly greater extent. These findings indicate that SPA may synergize with PARP inhibitors or DNA-damaging therapeutics (20). We determined that SPA-induced DNA damage occurs within hours, and the extent of DNA damage is correlated with AR overexpression and higher ligand levels, which in combination suppressed growth and induced senescence and apoptosis. These results are compatible with a mechanism of growth repression via transcriptionassociated AR-programmed double-strand DNA breaks $(19,21,61)$.

Our findings support a critical role for DNA-PKcs, a key kinase involved in NHEJ-mediated DNA repair, as a modulator of SPAinduced, AR-mediated DNA damage. In AR-overexpressing cells, we observed enhanced and persistent DNA-PKcs phosphorylation foci at S2056, which is important for DNA-PKcs activation and chromatin binding. SPA prolonged S2056 DNA-PKcs phosphorylation and impeded Thr2609 phosphorylation, an event that is ultimately required for DNA-PKcs dissociation from chromatin. These effects were amplified by cotreatment with the PARP inhibitor OLA. Our data suggest that retention of DNA-PKcs on chromatin in AR-overexpressing cells hinders completing the DNA repair process confirmed by an impaired DNA end-ligation assay, contributing to the elevated and persistent DNA damage induced by SPA. Notably, a previous study determined that AR splice variants (ARVs) could promote the repair of DNA damage induced by radiation via binding of ARVs to the catalytic subunit of DNA-PK (62). In contrast to ADT, which increases the expression of ARVs, SPA has been shown to repress the expression of ARVs (10), a result expected to compromise ARV-mediated DNA repair and promote growth arrest.

To further investigate the clinical context where SPA may be most efficacious, we evaluated tumors with BRCA2 loss and consequent HRD. We observed enhanced responses to SPA including accentuated DNA damage and apoptosis in such tumors. These findings are congruent with our clinical data showing associations between HR gene mutations and favorable responses to BAT. These findings support clinical studies of SPA in combination with PARP inhibitors, and trials evaluating this approach have recently been initiated and registered at ClinicalTrials.gov (NCT03516812). This work also supports clinical trials testing SPA in combination with DNA-PKcs inhibitors and in patients with PC with AR amplification or those who exhibit HR gene mutations.

\section{Methods}

Study design. The overall objective of this study was to determine the mechanism(s) by which supraphysiological concentrations of androgens (SPAs) repress PC growth. We used multiple PC cell lines and preclinical models with variable expression (de novo or engineered) of the AR and quantitated cellular responses across concentration ranges of androgens. We evaluated the effects of combining SPA with pharmacological inhibition of DNA repair pathway components including PARP and DNA-PKcs. We engineered cells to inactivate $B R C A 2$ and quantitated phenotypic responses to SPA relative to intact BRCA2. Standard methods and measurements were used to assess proliferation, apoptosis, senescence, DNA damage, and gene expression. A minimum of 3 biological replicates for each in vitro assay were obtained and are shown in the text and/or figure legends. Clinical samples were obtained from patients enrolled on studies of SPA and clinical-grade NextGen sequencing assays were used to determine germline or somatic mutations in genes known to mediate DNA repair. Clinical responses were determined by study personnel blinded to the results of DNA repair gene mutations.

Cell culture and treatment. PC cell lines PC3, LNCaP, VCaP, and DU145 were obtained from American Type Culture Collection (ATCC). The creation of PC3 $3^{\mathrm{AR}}$ and $\mathrm{LNCaP}{ }^{\mathrm{APIPC}}$ has previously been described $(15,31)$. LNCaP ${ }^{A R}$ were provided by Charles Sawyers. All cells were cultured as recommended by their suppliers and genotyped for authenticity by short tandem repeat analysis. All cells were confirmed negative for mycoplasma. DHT (Cayman Chemical), R1881 (Perkin Elmer), T (MilliporeSigma), OLA (Sellek Chemical), and the DNA-PKcs inhibitor Nu 7441 (Tocris Bioscience) were used in this study. Cells were treated with DHT, T, or R1881 and OLA simultaneously for 24 hours for confocal studies and 72 hours for cell death and survival assays. The derivation and propagation of the LuCaP PDX models were described previously (57). For ex vivo studies, LuCaP 35, 70, and 96CR PDX tumors were dissociated using Miltenyi Biotec's tumor dissociation kit, plated in 6-well dishes and treated with R1881, OLA, or the combination.

Confocal microscopy. Cells were plated on coverslips in 6-well dishes except for LuCaP 96CR, where coverslips were not used. After treatment, cells were fixed with 3.0\% paraformaldehyde for 20 minutes at room temperature, permeabilized with $0.2 \%$ Triton $\mathrm{X}-100$ in PBS for 10 minutes, and blocked in 3\% FBS in PBS containing $0.1 \%$ Triton X-100 for 1 hour. The coverslips were immunostained using $\gamma \mathrm{H} 2 \mathrm{AX}$ (Millipore), 53BP1, S2056 DNA PKcs, Thr2609 DNA-PKcs (Abcam), and RAD51 (Santa Cruz Biotechnology) primary antibodies, followed by fluorescently conjugated secondary antibodies (Invitrogen). When possible, cells were costained with multiple antibodies. Mounting and staining of the nuclei were performed using Vectashield (DAPI; Vector Laboratories). The number of foci from 70-100 cells were manually counted across multiple microscope fields. For each field, the average number of foci was determined per cell. The average of the fields was then plotted. Images were analyzed by Image J (National Institutes of Health).

Western blot. Cells or fragments of LuCaP xenografts were washed once in $1 \mathrm{x}$ PBS before lysis in 1\% SDS, $1 \%$ NP-40, $2 \%$ Tween-20, 1.5 M urea in PBS with protease inhibitors and phosphatase inhibitors obtained from Pierce Biotechnology. Lysates were collected with a cell scraper and boiled for 2 minutes. DNA was sheared by sonication. The lysates were subjected to immunoblot- 
ting as described (63) and probed with antibodies against S81AR (Santa Cruz Biotechnology), BRCA2 (MilliporeSigma), $\beta$-Tubulin (Sigma), AR, and GAPDH (both from Genetex).

Growth assays. Cell growth was assayed by plating 5000 cells per well in a tissue culture-treated 96-well black-sided, clear bottom plate (Corning) and allowed to adhere for 24 hours, then treated with R1881 or T, DHT, OLA, and/or Nu 7441 for 72 hours and assayed for apoptosis and viability using ApoLive Glo (Promega) following the manufacturer's instructions.

Cell-cycle analysis. Cell-cycle distribution was determined by flow cytometry for cells treated with R1881 and OLA for 72 hours. Cells were fixed in $70 \%$ ethanol and incubated in a solution containing propidium iodide $(50 \mathrm{mg} / \mathrm{mL})$, RNase A $(0.1 \mathrm{mg} / \mathrm{mL})$, Triton X $(0.05 \%)$, and analyzed on a Canton 2-2 flow cytometer (Beckton Dickinson). The raw data obtained were analyzed by Flowjo version 10 software. The results were normalized to control cells.

Cell synchronization by isoleucine deprivation. Isoleucine deprivation was performed by allowing the cells to become subconfluent and then replacing the media with isoleucine-depleted RPMI as previously described (32).

Comet assay. The comet assay silver staining kit (Trevigen) was used following the manufacturer's protocol as previously described (46). Image analysis and quantification were conducted via Image J (NIH). Tail moment and tail length were measured to quantify DNA damage. Tail moment $=$ percentage of DNA in the tail $\times$ tail length; in which the percentage of DNA in the tail $=$ tail area $\times$ tail average density $\times 100 /$ (tail area $\times$ tail average density) + (head area $\times$ head area intensity).

DNA end-ligation assay. One microgram of EcoRI-digested pUC19 DNA (as a surrogate for DSBs) was treated with nuclear extracts of LNCaP, LNCaP ${ }^{\mathrm{AR}}, \mathrm{PC} 3$, and $\mathrm{PC}^{\mathrm{AR}}$ cells (either untreated or treated with R1881 for 6 and 24 hours) in reaction buffer (46). The end-ligation mixtures were incubated at $37^{\circ} \mathrm{C}$ for 30 minutes to 1 hour and separated by electrophoresis on $0.6 \%$ agarose gels. Linearized EcoRI-digested pUC19 DNA with or without protein extract was used as negative control, and linearized pUC19 DNA treated with T4 DNA ligase was used as a positive control.

Senescence assay. The senescence assay was performed by using a senescence $\beta$-galactosidase staining kit (Cell Signaling) following the manufacturer's instructions. Cells were plated in 6-well plates 1 day before treatment with R1881 and/or OLA. After 3 days, cells were fixed and stained with staining solution containing X-gal (MilliporeSigma). The percentage of $\beta$-galactosidase-positive cells was determined by counting 5 different fields (approximately 70 cells/sample).

Whole transcriptome sequencing (RNA-Seq) and analysis. Biological replicate cultures of LNCaP cells grown in phenol-red free RPMI-1640 supplemented with $10 \%$ FBS were treated for 24 hours with media containing either 10\% FBS, $5 \%$ charcoal-stripped serum (ADT), or 5\% charcoal-stripped serum plus $100 \mathrm{nM}$ R1881 (SPA). RNA was isolated, sequenced, aligned, and analyzed as previously described (28). RNA sequencing data were deposited in the Gene Expression Omnibus database under the accession number GSE119598.

Ex vivo tissue culture and assays of treatment responses. We used gelatin or collagen sponges (Vetspon, MilliporeSigma) as a scaffold for culturing LuCaP 35CR and LuCaP 96 tumors that were dissected into slices 1- to $2-\mathrm{mm}^{3}$ thick, following published methods (64). After 24 hours, tissue slices were treated for 3 days continuously with $50 \mathrm{nM}$ $\mathrm{R} 1881$ or $25 \mu \mathrm{M}$ OLA or a combination. As indicated in the protocol, drug concentrations were 5-fold greater than standard tissue-culture concentrations. After 3 days, the tumor slices were placed in OCT (Fisher Healthcare) and flash frozen in liquid nitrogen. Frozen sections $(0.8 \mu \mathrm{m})$ were cut for staining. Confocal microscopy was performed by fixing tissue sections with paraformaldehyde after removing the OCT.

RNA collection and $q R T-P C R$. Total RNA was isolated from 6-well cell culture plates using an RNEasy kit (Qiagen) following the manufacturer's protocol. An Applied Biosystems 7900 sequence detector with SYBR Green PCR master mix (Invitrogen) was used for qRTPCR. PrimerQuest (IDT) was used to design primers, and reactions were normalized to the expression of the housekeeping gene RPL13A. A water negative control did not produce significant amplification products. Primer sequences were as follows: AR 5'-GAATGAGGCACCTCTCTCAAG-3', 5'-CAGCCCATCCACTGGAATAA-3'; KLK3 5'-GCATGGGATGGGGATGAAGTAAG-3', 5'-CATCAAATCTGAGGGTTGTCTGGA-3'; RPL13a 5'-CCTGGAGGAGAAGAGGAAAGA-3', 5'-TTGAGGACCTCTGTGTATTTG-3'; NKX3.1 5'-ACTAATGAGGTACGCTGAGGC-3', $\quad$ 5'-TGGCCAACTTTCTATTAACTTATG-3'; TMPRSS2 5'-CATGATCTGTGCCGGCTTCCTGCAGG-3', 5'-CTTGTATCCCCTATCAGCCACCAGATA-3'.

Establishment of BRCA2-deficient LNCaP cell lines. We established 2 LNCaP cell line models with BRCA2 deficiency. We constructed an inducible BRCA2 knockdown model using a doxycycline/tetracycline system. A Tet-shBRCA2 construct was generated by cloning the sequence GGGAAACACTCAGATTAAA_TGACTAGT_TTTAATCTGAGTGTTTCCC_TTTTTT (65) into the EZ-Tet-pLKO-Hygro vector (Addgene plasmid 85972) as previously described (66). LNCaP cells were infected with lentivirus and selected with $500 \mu \mathrm{g} / \mathrm{mL}$ hygromycin. Knockdown was induced by the addition of $100 \mathrm{ng} / \mathrm{mL}$ doxycycline to media for at least 96 hours. Samples were lysed with RIPA lysis buffer and normalized by BCA assay (Pierce Biotechnology). Lysates were run on $3 \%-8 \%$ tris acetate SDS gels (Invitrogen) and transferred onto Immoblin-FL PVDF membrane (MilliporeSigma). Primary antibodies included BRCA2 (Cell Signaling Technologies, 10741, 1:2000) and hFab-Rhodamine-Tubulin (BioRad, 12004165, 1:5000). Secondary antibody includes StarBright520-goat-anti-rabbit (BioRad, $12005869,1: 5000)$. Antibodies were diluted in blocking buffer (5\% $\mathrm{BSA} /$ mixture of TBS and polysorbate 20). Images were acquired on a BioRad ChemiDocMP fluorescence imaging system.

We used a CRISPR/Cas9 approach to delete BRCA2 in LNCaP cells. To create the sgRNA targeting $B R C A 2$, an sgRNA protospacer of GAAACCATCTTATAATCAGC was cloned in to the ESP3I enzyme (Thermo Fisher Scientific) sites of the lentivirus expression vector lentiCRISPRv2 (Addgene plasmid 52961) (67) using annealed oligos plus BRCA2_sgRNA ${ }^{+}$(caccgGAAACCATCTTATAATCAGC) and BRCA2 sgRNA- (aaacGCTGATTATAAGATGGTTTCc). LNCaPs were transduced with the BRCA2 sgRNA CRISPR vector and selected for 5 days with puromycin. They were then plated 1 cell/well in 96-well plates. Resulting colonies were screened by Western blot for loss of BRCA2 expression using an antibody from MilliporeSigma and a particular isolate (B11) was chosen for the experiment. Further analyses of isolate $\mathrm{B} 11$, designated here as $\mathrm{LNCaP}^{\mathrm{BRCA} 2}$, demonstrated a mixed-heterozygous population comprising cells with WT sequence and cells with 102 bp or 21 bp deletions detected.

Clinical study design. The primary goal of the clinical study was to evaluate the genomic discriminators of response/resistance to SPA using an approach administering monthly high-dose $\mathrm{T}$ (BAT) in men 
with CRPC. Supraphysiological T alternates with low or near-castrate levels. We obtained biospecimens from patients with CRPC enrolled into 2 ongoing clinical trials testing BAT. The clinical studies were approved by IRBs of Johns Hopkins University and the University of Washington/Fred Hutchinson Cancer Research Center. Informed consent was obtained after the nature and possible consequences of the studies were explained. The first study was a phase II trial testing BAT following progression on either enzalutamide (cohort 1), abiraterone (cohort 2), or medical/surgical castration alone (cohort 3), registered at ClinicalTrials.gov (NCT02090114) (11). Following progression on BAT, patients were rechallenged with the agent they received before enrolling (i.e., enzalutamide, abiraterone, or ADT). The coprimary endpoint was $\mathrm{PSA}_{50}$ response (i.e., $\geq 50 \%$ decline in PSA from baseline) following treatment with BAT and following rechallenge with enzalutamide (cohort 1), abiraterone (cohort 2), or ADT (cohort 3) after progressing on BAT. The second study is a phase II randomized trial testing BAT versus enzalutamide in patients who had previously progressed on abiraterone, registered at ClinicalTrials. gov (NCT02286921). The primary endpoint of this study is clinical/ radiographic progression. Both trials were approved by the Johns Hopkins IRB. Additional biospecimens were obtained at the University of Washington per separate blood/tissue collection protocols approved by the Fred Hutchinson Cancer Research Center/University of Washington Cancer Consortium IRB.

In both clinical trials, BAT was administered as an i.m. injection of either T cypionate or enanthate $400 \mathrm{mg}$ every 28 days. Subjects were also maintained on ADT in order to suppress endogenous gonadal androgen synthesis. T cypionate and enanthate have identical pharmacokinetics and have been shown to produce supraphysiologic T levels (>1500 ng/dL) within a few days of the injection followed by a decline in $\mathrm{T}$ levels to the near-castrate range by day 28 in patients maintained on $\operatorname{ADT}(10,68)$. Samples underwent germline or somatic NGS, and a variety of clinical-grade NGS platforms were used, including UW-OncoPlex $(n=75)$, PlasmaSELECT (Personal Genome Diagnostics, Inc.) ( $n=12)$, FoundationOne CDX (Foundation Medicine, Inc.) $(n=11)$, Color (Color Genomics) ( $n=10)$, and Guardant360 (Guardant Health, Inc.) $(n=1)(69)$. One additional case was sequenced as part of the SU2C/PCF International Dream Team study as previously described (56). Given the concern for false negatives, cases were excluded if plasma-based NGS did not reveal a somatic alteration. Absence of a germline alteration was not assumed to indicate absence of somatic alterations. Associations between $\mathrm{PSA}_{50}$ response to BAT and mutations in genes of interest (i.e., DNA repair genes) were evaluated and differences were sought using a $\chi^{2}$ test.

Statistics. Two-tailed Student's $t$ test or a 2-way ANOVA followed by Tukey's multiple comparison test were used to compare significance between grouped quantitative data sets (e.g., qRT-PCR, foci number, growth, and caspase activity data) using GraphPad Prism 8.0 software. At least 3 replicates were used for each experimental group. Each experiment was analyzed in total and the presented statistical significance in the graphs represents this analysis. Differences were considered significant if $P$ is less than or equal to 0.05 .

Study approval. The clinical studies were approved by IRBs of the Johns Hopkins University and the University of Washington/ Fred Hutchinson Cancer Research Center. Informed consent was obtained after the nature and possible consequences of the studies were explained.

\section{Author contributions}

PC, MTS, JML, SRD, and PSN designed the research. PC, JML, IC, MDN, SBF, RT, EM, JL, CCP, HML, EC, and ESA performed the research. PC, MTS, JML, and IC analyzed the data. PC and PSN wrote the manuscript and all authors edited and approved the final manuscript. The order of equal contributing co-first authors was determined by drawing lots. The order of co-senior authors was determined alphabetically.

\section{Acknowledgments}

We are grateful to the patients who participated in these studies. We acknowledge the Fred Hutch Scientific Imaging Shared Resource for assistance with imaging experiments. We thank Holly Nguyen, Lisha Brown, and Lisa Ang for their technical support involving LuCaP tumor studies. We gratefully acknowledge research support from Fred Hutch/University of Washington Cancer Consortium grant P30CA015704-40, NIH P50CA97186, R21CA194798, P01CA163227, and Congressionally Directed Medical Research Program (CDMRP) Awards PC170503 and PC170431. PC was supported by a CDMRP postdoctoral fellowship award W81XWH-15-1-0535. MDN was supported by a CDMRP postdoctoral fellowship award W81XWH-16-1-0206. MTS was supported by a Prostate Cancer Foundation Young Investigator Award and a Department of Defense (DOD) award W81XWH-16-1-0484. JL is supported by National Cancer Institute CA185297 and a DOD CDMRP award W81XWH-15-2-0050. ESA is partially supported by NIH grants P30CA006973 and R01 CA185297 and CDMRP grant W81XWH-16-PCRP-CCRSA. CCP was supported by DOD awards PC170510, PC170503P2, and PC141019.

Address correspondence to: Samuel R. Denmeade, Department of Oncology, The Johns Hopkins University School of Medicine, $201 \mathrm{~N}$. Broadway, Baltimore, Maryland 21287, USA. Phone: 410.955.8875; Email: denmesa@jhmi.edu. Or to: Peter S. Nelson, Division of Human Biology, Fred Hutchinson Cancer Research Center, 1100 Fairview Avenue N, Mailstop D4-100, Seattle, Washington 981091024, USA. Phone: 206.667.3377; Email:pnelson@fredhutch.org.
1. Cunha GR, et al. The endocrinology and developmental biology of the prostate. Endocr Rev. 1987;8(3):338-362.

2. Bosland MC. The role of steroid hormones in prostate carcinogenesis. J Natl Cancer Inst Monographs. 2000;(27):39-66.

3. Debes JD, Tindall DJ. The role of androgens and the androgen receptor in prostate cancer. Cancer Lett. 2002;187(1-2):1-7.
4. Scher HI, Sawyers CL. Biology of progressive, castration-resistant prostate cancer: directed therapies targeting the androgen-receptor signaling axis. J Clin Oncol. 2005;23(32):8253-8261.

5 . Mohler JL, et al. Androgen and glucocorticoid receptors in the stroma and epithelium of prostatic hyperplasia and carcinoma. Clin Cancer Res. 1996;2(5):889-895.

6. Hobisch A, Culig Z, Radmayr C, Bartsch G,
Klocker H, Hittmair A. Androgen receptor status of lymph node metastases from prostate cancer. Prostate. 1996;28(2):129-135.

7. Sadi MV, Walsh PC, Barrack ER. Immunohistochemical study of androgen receptors in metastatic prostate cancer. Comparison of receptor content and response to hormonal therapy. Cancer. 1991;67(12):3057-3064.

8. Kokontis JM, Hay N, Liao S. Progression of 
LNCaP prostate tumor cells during androgen deprivation: hormone-independent growth, repression of proliferation by androgen, and role for p27Kip1 in androgen-induced cell cycle arrest. Mol Endocrinol. 1998;12(7):941-953.

9. Umekita Y, Hiipakka RA, Kokontis JM, Liao S. Human prostate tumor growth in athymic mice: inhibition by androgens and stimulation by finasteride. Proc Natl Acad Sci USA. 1996;93(21):11802-11807.

10. Schweizer MT, et al. Effect of bipolar androgen therapy for asymptomatic men with castration-resistant prostate cancer: results from a pilot clinical study. Sci Transl Med. 2015;7(269):269ra2.

11. Teply BA, et al. Bipolar androgen therapy in men with metastatic castration-resistant prostate cancer after progression on enzalutamide: an open-label, phase 2, multicohort study. Lancet Oncol. 2018;19(1):76-86.

12. Geck P, Maffini MV, Szelei J, Sonnenschein C, Soto AM. Androgen-induced proliferative quiescence in prostate cancer cells: the role of AS3 as its mediator. Proc Natl Acad Sci USA. 2000;97(18):10185-10190.

13. Reddel RR, Sutherland RL. Effects of pharmacological concentrations of estrogens on proliferation and cell cycle kinetics of human breast cancer cell lines in vitro. Cancer Res. 1987;47(20):5323-5329.

14. Song RX, et al. Effect of long-term estrogen deprivation on apoptotic responses of breast cancer cells to 17beta-estradiol. J Natl Cancer Inst. 2001;93(22):1714-1723.

15. Kumar A, et al. Substantial interindividual and limited intraindividual genomic diversity among tumors from men with metastatic prostate cancer. Nat Med. 2016;22(4):369-378.

16. Chuu CP, et al. Androgen suppresses proliferation of castration-resistant LNCaP 104-R2 prostate cancer cells through androgen receptor, Skp2, and c-Myc. Cancer Sci. 2011;102(11):2022-2028.

17. Litvinov IV, et al. Androgen receptor as a licensing factor for DNA replication in androgen-sensitive prostate cancer cells. Proc Natl Acad Sci USA. 2006;103(41):15085-15090.

18. Mohammad OS, et al. Supraphysiologic testosterone therapy in the treatment of prostate cancer: models, mechanisms and questions. Cancers (Basel). 2017;9(12):E166.

19. Haffner MC, et al. Androgen-induced TOP2B-mediated double-strand breaks and prostate cancer gene rearrangements. Nat Genet. 2010;42(8):668-675.

20. Hedayati M, et al. Androgen deprivation followed by acute androgen stimulation selectively sensitizes AR-positive prostate cancer cells to ionizing radiation. Clin Cancer Res. 2016;22(13):3310-3319.

21. Ju BG, et al. A topoisomerase IIbeta-mediated dsDNA break required for regulated transcription. Science. 2006;312(5781):1798-1802.

22. Ju BG, Rosenfeld MG. A breaking strategy for topoisomerase IIbeta/PARP-1-dependent regulated transcription. Cell Cycle. 2006;5(22):2557-2560.

23. Polkinghorn WR, et al. Androgen receptor signaling regulates DNA repair in prostate cancers. Cancer Discov. 2013;3(11):1245-1253.

24. Goodwin JF, et al. A hormone-DNA repair circuit governs the response to genotoxic insult. Cancer Discov. 2013;3(11):1254-1271.

25. Gao S, et al. Androgen receptor tumor suppressor function is mediated by recruitment of retinoblastoma protein. Cell Rep. 2016;17(4):966-976.

26. Liao S, Liang T, Fang S, Castañeda E, Shao TC. Steroid structure and androgenic activity. Specificities involved in the receptor binding and nuclear retention of various androgens. J Biol Chem. 1973;248(17):6154-6162.

27. Snaterse G, Visser JA, Arlt W, Hofland J. Circulating steroid hormone variations throughout different stages of prostate cancer. Endocr Relat Cancer. 2017;24(11):R403-R420.

28. Bluemn EG, et al. Androgen receptor pathway-independent prostate cancer is sustained through FGF signaling. Cancer Cell. 2017;32(4):474-489.e6.

29. Kemppainen JA, Lane MV, Sar M, Wilson EM. Androgen receptor phosphorylation, turnover, nuclear transport, and transcriptional activation. Specificity for steroids and antihormones. J Biol Chem. 1992;267(2):968-974.

30. Brown TR, Rothwell SW, Migeon CJ. Comparison of methyltrienolone and dihydrotestosterone binding and metabolism in human genital skin fibroblasts. JSteroid Biochem. 1981;14(10):1013-1022.

31. Kaufman M, Pinsky L. The dissociation of testosterone- and 5 alpha-dihydrotestosterone-receptor complexes formed within cultured human genital skin fibroblasts. J Steroid Biochem. 1983;18(2):121-125.

32. Cifuentes E, Croxen R, Menon M, Barrack ER, Reddy GP. Synchronized prostate cancer cells for studying androgen regulated events in cell cycle progression from G1 into S phase. JCell Physiol. 2003;195(3):337-345.

33. Traish AM, Williams DF, Hoffman ND, Wotiz HH. Validation of the exchange assay for the measurement of androgen receptors in human and dog prostates. Prog Clin Biol Res. 1988;262:145-160.

34. Menon M, Tananis CE, Hicks LL, Hawkins EF, McLoughlin MG, Walsh PC. Characterization of the binding of a potent synthetic androgen, methyltrienolone, to human tissues. J Clin Invest. 1978;61(1):150-162.

35. Walsh PC, Greco JM, Tananis CE, Hicks LL, McLoughlin MG, Menon M. The binding of a potent synthetic androgen - methyltrienolone (R 1881) - to cytosol preparations of human prostatic cancer. Trans Am Assoc Genitourin Surg. 1977;69:78-80.

36. Gregory CW, Johnson RT, Mohler JL, French FS, Wilson EM. Androgen receptor stabilization in recurrent prostate cancer is associated with hypersensitivity to low androgen. Cancer Res. 2001;61(7):2892-2898

37. Wilson EM, French FS. Binding properties of androgen receptors. Evidence for identical receptors in rat testis, epididymis, and prostate. J Biol Chem. 1976;251(18):5620-5629.

38. Grino PB, Griffin JE, Wilson JD. Testosterone at high concentrations interacts with the human androgen receptor similarly to dihydrotestosterone. Endocrinology. 1990;126(2):1165-1172.

39. Syms AJ, Norris JS, Panko WB, Smith RG. Mechanism of androgen-receptor augmentation. Analysis of receptor synthesis and degradation by the density-shift technique. J Biol Chem. 1985;260(1):455-461.

40. Chen S, Gulla S, Cai C, Balk SP. Androgen receptor serine 81 phosphorylation mediates chromatin binding and transcriptional activation. J Biol Chem. 2012;287(11):8571-8583.

41. Chen S, Xu Y, Yuan X, Bubley GJ, Balk SP. Androgen receptor phosphorylation and stabilization in prostate cancer by cyclin-dependent kinase 1. Proc Natl Acad Sci USA. 2006;103(43):15969-15974.

42. Gioeli D, et al. Androgen receptor phosphorylation. Regulation and identification of the phosphorylation sites. J Biol Chem. 2002;277(32):29304-29314.

43. Nelson PS, et al. The program of androgen-responsive genes in neoplastic prostate epithelium. Proc Natl Acad Sci USA. 2002;99(18):11890-11895.

44. Bolla M, et al. Improved survival in patients with locally advanced prostate cancer treated with radiotherapy and goserelin. $N$ Engl JMed. 1997;337(5):295-300.

45. Bolla $\mathrm{M}$, et al. Long-term results with immediate androgen suppression and external irradiation in patients with locally advanced prostate cancer (an EORTC study): a phase III randomised trial. Lancet. 2002;360(9327):103-106.

46. Chatterjee P, Plesca D, Mazumder S, Boutros J, Yannone SM, Almasan A. Defective chromatin recruitment and retention of NHEJ core components in human tumor cells expressing a Cyclin $\mathrm{E}$ fragment. Nucleic Acids Res. 2013;41(22):10157-10169.

47. Lord CJ, Tutt AN, Ashworth A. Synthetic lethality and cancer therapy: lessons learned from the development of PARP inhibitors. Annu Rev Med. 2015;66:455-470.

48. Mao Z, Bozzella M, Seluanov A, Gorbunova V. DNA repair by nonhomologous end joining and homologous recombination during cell cycle in human cells. Cell Cycle. 2008;7(18):2902-2906.

49. Joly-Pharaboz MO, et al. Androgen inhibits the growth of carcinoma cell lines established from prostate cancer xenografts that escape androgen treatment. JSteroid Biochem Mol Biol. 2008;111(1-2):50-59.

50. Kokontis JM, et al. Androgen suppresses the proliferation of androgen receptor-positive castration-resistant prostate cancer cells via inhibition of Cdk2, CyclinA, and Skp2. PLoS ONE. 2014;9(10):e109170.

51. Cui X, Yu Y, Gupta S, Cho YM, Lees-Miller SP, Meek K. Autophosphorylation of DNAdependent protein kinase regulates DNA end processing and may also alter double-strand break repair pathway choice. Mol Cell Biol. 2005;25(24):10842-10852.

52. Chen BP, et al. Ataxia telangiectasia mutated (ATM) is essential for DNA-PKcs phosphorylations at the Thr-2609 cluster upon DNA double strand break. JBiol Chem. 2007;282(9):6582-6587.

53. Meek K, Douglas P, Cui X, Ding Q, Lees-Miller SP. Trans autophosphorylation at DNA-dependent protein kinase's two major autophosphorylation site clusters facilitates end processing but not end joining. Mol Cell Biol. 2007;27(10):3881-3890.

54. Dobbs TA, Tainer JA, Lees-Miller SP. A structural model for regulation of NHEJ by DNA-PKcs autophosphorylation. DNA Repair (Amst). 2010;9(12):1307-1314. 
55. Kukolj E, Kaufmann T, Dick AE, Zeillinger R, Gerlich DW, Slade D. PARP inhibition causes premature loss of cohesion in cancer cells. Oncotarget. 2017;8(61):103931-103951.

56 . Robinson $\mathrm{D}$, et al. Integrative clinical genomics of advanced prostate cancer. Cell. 2015;161(5):1215-1228.

57. Nguyen HM, et al. LuCaP prostate cancer patient-derived xenografts reflect the molecular heterogeneity of advanced disease and serve as models for evaluating cancer therapeutics. Prostate. 2017;77(6):654-671.

58. Morris MJ, et al. Phase 1 trial of high-dose exogenous testosterone in patients with castrationresistant metastatic prostate cancer. Eur Urol. 2009;56(2):237-244.

59. Szmulewitz R, et al. A randomized phase 1 study of testosterone replacement for patients with low-risk castration-resistant prostate cancer. Eur
Urol. 2009;56(1):97-103.

60. Kumar A, et al. Substantial interindividual and limited intraindividual genomic diversity among tumors from men with metastatic prostate cancer. Nat Med. 2016;22(4):369-378.

61. Puc J, Aggarwal AK, Rosenfeld MG. Physiological functions of programmed DNA breaks in signal-induced transcription. Nat Rev Mol Cell Biol. 2017;18(8):471-476.

62. Yin Y, et al. Androgen receptor variants mediate DNA repair after prostate cancer irradiation. Cancer Res. 2017;77(18):4745-4754.

63. Huber RM, et al. DNA damage induces GDNF secretion in the tumor microenvironment with paracrine effects promoting prostate cancer treatment resistance. Oncotarget. 2015;6(4):2134-2147.

64. Centenera MM, Raj GV, Knudsen KE, Tilley WD, Butler LM. Ex vivo culture of human prostate tissue and drug development. Nat Rev Urol.
2013;10(8):483-487.

65. Zimmer J, et al. Targeting BRCA1 and BRCA2 deficiencies with G-quadruplex-interacting compounds. Mol Cell. 2016;61(3):449-460.

66. Frank SB, Schulz VV, Miranti CK. A streamlined method for the design and cloning of shRNAs into an optimized Dox-inducible lentiviral vector. BMC Biotechnol. 2017;17(1):24.

67. Sanjana NE, Shalem O, Zhang F. Improved vectors and genome-wide libraries for CRISPR screening. Nat Methods. 2014;11(8):783-784.

68. Nieschlag ES, Behre HM. Testosterone: Action, Deficiency, Substitution. Cambridge, UK: Cambridge University Press; 2012.

69. Pritchard CC, et al. Validation and implementation of targeted capture and sequencing for the detection of actionable mutation, copy number variation, and gene rearrangement in clinical cancer specimens. JMol Diagn. 2014;16(1):56-67. 